\title{
Splitting of separatrices in Hamiltonian systems with one and a half degrees of freedom
}

\author{
Amadeu Delshams and Tere M. Seara \\ Departament de Matemàtica Aplicada I \\ Universitat Politècnica de Catalunya \\ Diagonal 647, 08028 Barcelona, Spain
}

\begin{abstract}
The splitting of separatrices for Hamiltonians with $1 \frac{1}{2}$ degrees of freedom

$$
h(x, t / \varepsilon)=h^{0}(x)+\mu \varepsilon^{p} h^{1}(x, t / \varepsilon)
$$

is measured. We assume that $h^{0}(x)=h^{0}\left(x_{1}, x_{2}\right)=x_{2}^{2} / 2+V\left(x_{1}\right)$ has a separatrix $x^{0}(t), h^{1}(x, \theta)$ is $2 \pi$-periodic in $\theta, \mu$ and $\varepsilon>0$ are independent small parameters, and $p \geq 0$. Under suitable conditions of meromorphicity for $x_{2}^{0}(u)$ and the perturbation $h^{1}\left(x^{0}(u), \theta\right)$, the order $\ell$ of the perturbation on the separatrix is introduced, and it is proved that, for $p \geq \ell$, the splitting is exponentially small in $\varepsilon$, and is given in first order by the Melnikov function.
\end{abstract}

Keywords: homoclinic solutions, normal forms, Melnikov method, averaging method 


\section{Introduction and main results}

In his celebrated memoir on the three-body problem [Poi90], Poincare introduced the phenomenon of the splitting of the separatrices (transversal intersection of invariant manifolds that are coincident for the unperturbed problem), as the main obstruction for the integrability of the differential equations. To measure the size of such splitting, he developed a perturbative method in the parameter of perturbation, which was rediscovered 70 years later, and is now well-known as the Melnikov method. At that time, he was already aware that the size of the splitting of the separatrices predicted by his perturbative method was exponentially small with respect to the parameter of perturbation $\varepsilon$ [Poi90, page 223], a fact which prevented him to provide rigorous results, since the remainder of his perturbative expansion was, in principle, $\mathrm{O}\left(\varepsilon^{2}\right)$.

It was not until the last decade that an effective measure of such exponentially small splitting of separatrices was addressed by several authors [HMS88, GLT91, Ang93], and complete proofs of its asymptotic behavior were finally obtained for the rapidly forced pendulum in some papers [DS92, Gel93, EKS93, Tre97, Sau95], for different kind of hypotheses on the size of the perturbative forcing.

For more general systems, upper estimates were obtained in several papers [Nei84, Fon93, Sim94, Fon95, FS96], and only very recently, asymptotic expressions or lower estimates have been justified for some relevant examples [Gal94, Gel95, DGJS97, RW97b].

The aim of this paper is to expound a general method to validate the exponentially small expressions provided by the Melnikov function for general Hamiltonian systems with $1 \frac{1}{2}$ degrees of freedom and a rapidly oscillatory dependence in time. In particular, we are not going to restrict ourselves, like most of the cited authors, to the simpler reversible second order equations, for which the results contained in [DS92] for the rapidly forced pendulum - the standard paradigm—are readily adaptable.

To deal with systems of this generality, we introduce suitable flow-box canonical coordinates, in such a way that the perturbed invariant manifolds take a simple form, as well as their associated dynamics. These canonical flow-box coordinates provide an invariant measure for the splitting of separatrices, and can also be applied in a broader setting. We also rely on an extension theorem, a tool already introduced by the authors in [DS92], which permits to get a priori sharp estimates for the allowed size of the perturbation.

The main ideas of this paper were already announced in [DS93, DS94]. However, these papers were not developed in their outmost generality, and because of this, we now proceed to explain the method with full details, and all the required hypotheses will be thoroughly discussed. We hope that this detailed and fully rigorous account of a fairly general framework will provide a firm starting point for those who want to enter the field and serve as a solid foundation for future work.

We shall consider a Hamiltonian of the form

$$
h(x, t / \varepsilon)=h^{0}(x)+\mu \varepsilon^{p} h^{1}(x, t / \varepsilon)
$$


where $h^{0}(x)=h^{0}\left(x_{1}, x_{2}\right)=x_{2}^{2} / 2+V\left(x_{1}\right), \mu$ and $\varepsilon>0$ are independent small parameters, and $p \geq 0$.

The Hamiltonian system associated to $h$ is a perturbation of the Hamiltonian system associated to $h^{0}$ :

$$
\dot{x}_{1}=x_{2}, \quad \dot{x}_{2}=f\left(x_{1}\right),
$$

where $f\left(x_{1}\right)=-V^{\prime}\left(x_{1}\right)$, which can be written also as a second order equation

$$
\ddot{x}_{1}=f\left(x_{1}\right) \text {. }
$$

We will assume the following hypotheses:

H1 The unperturbed system (1.1) has a saddle point at the origin with characteristic exponents $\pm \omega^{0}$, with $\omega^{0}>0$, and there exists a homoclinic solution $x^{0}(t)=$ $\left(x_{1}^{0}(t), x_{2}^{0}(t)\right)$ to this point: $x^{0}(t) \longrightarrow 0$ for $t \rightarrow \pm \infty$. This solution is commonly referred as a separatrix.

H2 The function $f\left(x_{1}\right)$ is real entire, and $x_{2}^{0}(u)=\dot{x}_{1}^{0}(u)$ is analytic on a strip $|\Im u|<a$, with a pole of order $r$ at $u= \pm a \mathrm{i}$ as its only singularity on each line $\Im u= \pm a \mathrm{i}$.

H3 The function $h^{1}(x, \theta)$ is $2 \pi$-periodic and $\mathcal{C}^{1}$ in $\theta$, with zero mean: $\int_{0}^{2 \pi} h^{1}(x, \theta) \mathrm{d} \theta=$ 0 . With respect to $x$, it can take either of the following forms:

(a) if $f$ is $2 \pi$-periodic, $h^{1}$ is a trigonometric polynomial in $x_{1}$ and a polynomial in $x_{2} ; h^{1}(x, \theta)=x_{1} g(\theta)$ is also allowed,

(b) $h^{1}$ is a polynomial in $x$, in the case that $f$ is not $2 \pi$-periodic.

As a consequence of Hypothesis $H 3, h^{1}(x, \theta)$ can be written as a sum of monomials in the variable $x$, each of which has a pole at $u= \pm a \mathrm{i}$, when $x=x^{0}(u)$, for every $\theta$. We will denote by $\ell$ the greatest order of this pole among these monomials, and we will call it the order of the perturbation on the separatrix.

To state our results, let us begin by writing the Hamiltonian system associated to $h$ :

$$
\begin{aligned}
& \dot{x}_{1}=x_{2}+\mu \varepsilon^{p} \partial_{2} h^{1}(x, t / \varepsilon), \\
& \dot{x}_{2}=f\left(x_{1}\right)-\mu \varepsilon^{p} \partial_{1} h^{1}(x, t / \varepsilon) .
\end{aligned}
$$

Due to the fact that system (1.2) is $2 \pi \varepsilon$-periodic in time, we can consider the associated Poincaré map defined by:

$$
P\left(x_{0}\right)=x(2 \pi \varepsilon),
$$

where $x(t)$ is the solution of system (1.2) that begins at $x_{0}$ when $t=0$.

For $\mu=0$, system (1.2) becomes autonomous and therefore the phase portraits of the Poincaré map $P$ and system (1.1) are identical. In fact, this phase portrait is foliated by the level curves of the Hamiltonian $h^{0}$. If we assume, without loss of generality, $V(0)=0$, the homoclinic orbit $x^{0}$ is contained in the level curve $h^{0}(x)=0$. 
For $\mu \neq 0$ but small enough, the dynamics of system (1.2) becomes more intricate and the phase portrait of the Poincaré map $P$ changes. There exists a hyperbolic fixed point near $(0,0)$ whose stable and unstable curves intersect $-P$ is an area preserving map-but generically they do not coincide. We denote $x^{h}:=\left(x_{1}^{h}, x_{2}^{h}\right)$ the homoclinic point that is closest to the unperturbed one $x^{0}(0)$.

The main goal of this paper is to give an asymptotic formula for the area $A$ of the lobe that remains between the two invariant curves from $x^{h}$ to their next intersection, as well as the angle $\alpha$ between the invariant curves at $x^{h}$. Our results are summarized in the next theorem.

Theorem 1.1 (Main Theorem) Under hypotheses $H 1-H 3$, assume that $\gamma:=p-\ell \geq$ 0 . Then, for $\varepsilon \rightarrow 0^{+}, \mu \rightarrow 0$, the following formulae hold:

$$
\begin{aligned}
A & =\mu \varepsilon^{p}\left[\int_{s_{0}}^{\bar{s}_{0}} M(\sigma, \varepsilon) \mathrm{d} \sigma\right]+\mathrm{O}\left(\mu^{2} \varepsilon^{2 \gamma+r}, \mu \varepsilon^{p+2}\right) \mathrm{e}^{-a / \varepsilon}, \\
\sin \alpha & =\mu \varepsilon^{p} \frac{M^{\prime}\left(s_{0}, \varepsilon\right)}{\left\|x^{0}\left(s_{0}\right)\right\|^{2}}+\mathrm{O}\left(\mu^{2} \varepsilon^{2 \gamma+r-2}, \mu \varepsilon^{p}\right) \mathrm{e}^{-a / \varepsilon},
\end{aligned}
$$

where $s_{0}<\bar{s}_{0}$ are the two zeros of the Melnikov function

$$
M(s, \varepsilon)=\int_{-\infty}^{+\infty}\left\{h^{0}, h^{1}\right\}\left(x^{0}(t+s), t / \varepsilon\right) \mathrm{d} t=\sum_{k \neq 0} M_{k}(\varepsilon) \mathrm{e}^{\mathrm{i} k s / \varepsilon},
$$

closest to zero.

This theorem gives upper estimates of exponentially small order for the area $A$ and the angle $\alpha$. We now introduce an additional hypothesis on the Poisson bracket of $h^{0}$ and $h^{1}$ over $x^{0}(u)$ :

$H 4 J_{ \pm 1}\left(x^{0}(u)\right)$ has a pole of order exactly $\ell+1$ at $u= \pm a \mathrm{i}$, where

$$
J(x, \theta):=\left\{h^{0}, h^{1}\right\}(x, \theta)=\sum_{k \neq 0} J_{k}(x) \mathrm{e}^{\mathrm{i} k \theta} .
$$

In other words, if one considers the Laurent expansion $J_{ \pm 1}\left(x^{0}(u)\right)=\sum_{k<\ell+1} J_{ \pm 1, k}(u \mp$ $a \mathrm{i})^{-k}$ of $J_{ \pm 1}\left(x^{0}(u)\right)$ at $u= \pm a \mathrm{i}$, hypothesis $H 4$ is equivalent to assume that the coefficient $J_{1, \ell+1}=\overline{J_{-1, \ell+1}}$ is not zero. Under this generic additional hypothesis, a direct computation of the Melnikov function shows that Theorem 1.1 provides asymptotic expressions:

Corollary 1.2 If moreover hypothesis $H 4$ holds, the first terms of $A$ and $\sin \alpha$ in Theorem 1.1-those containing the Melnikov function (1.4)-are not zero and are dominant with respect to the second ones, for $\varepsilon \rightarrow 0^{+}, \mu \rightarrow 0$ :

$$
\begin{aligned}
A & =4\left|J_{1, \ell+1}\right| \mu \varepsilon^{\gamma+1} \mathrm{e}^{-a / \varepsilon}\left[1+\mathrm{O}\left(\mu \varepsilon^{\gamma+r-1}, \varepsilon^{\ell+1}\right)\right], \\
\sin \alpha & =-\frac{2\left|J_{1, \ell+1}\right|}{\left\|x^{0}(0)\right\|^{2}} \mu \varepsilon^{\gamma-1} \mathrm{e}^{-a / \varepsilon}\left[1+\mathrm{O}\left(\mu \varepsilon^{\gamma+r-1}, \varepsilon\right)\right] .
\end{aligned}
$$


Let us now discuss some examples satisfying hypotheses $H 1-H 4$ :

1. A forced pendulum equation, with Hamiltonian

$$
h=\frac{x_{2}^{2}}{2}-\left(\cos x_{1}+1\right)+\mu \varepsilon^{p} x_{2} \cos x_{1} \sin \frac{t}{\varepsilon} .
$$

For $\mu=0$, this system has homoclinic orbits $\Gamma_{ \pm}=\left\{\left(x_{1}^{0}(t), \pm x_{2}^{0}(t)\right)\right\}$, where $x_{1}^{0}(t)=$ $2 \arctan (\sinh t), x_{2}^{0}(u)=\dot{x}_{1}^{0}(u)$ has poles of order $r=1$ at $u= \pm \pi \mathrm{i} / 2$, and $\ell=3$.

2. A perturbed Duffing equation, with Hamiltonian

$$
h=\frac{x_{2}^{2}}{2}-\frac{x_{1}^{2}}{2}+\frac{x_{1}^{4}}{4}+\mu \varepsilon^{p}\left(\frac{x_{2}^{2}}{2} \cos \frac{t}{\varepsilon}+x_{1} x_{2} \sin \frac{2 t}{\varepsilon}\right) .
$$

For $\mu=0$, the homoclinic orbits are $\Gamma_{ \pm}=\left\{\left( \pm x_{1}^{0}(t), x_{2}^{0}(t)\right)\right\}$, where $x_{1}^{0}(t)=$ $\sqrt{2} / \cosh t, x_{2}^{0}(u)=\dot{x}_{1}^{0}(u)$ has poles of order $r=2$ at $u= \pm \pi \mathrm{i} / 2$, and $\ell=4$.

3. A perturbed cubic potential equation, with Hamiltonian

$$
h=\frac{x_{2}^{2}}{2}-\frac{x_{1}^{2}}{2}+\frac{x_{1}^{3}}{3}+\mu \varepsilon^{p} x_{1} \cos \frac{t}{\varepsilon} .
$$

For $\mu=0$, this system has the homoclinic orbit $\Gamma=\left\{\left(x_{1}^{0}(t), x_{2}^{0}(t)\right)\right\}$, where $x_{1}^{0}(t)=$ $(\sqrt{3} / 2)(\cosh (t / 2))^{-2}, x_{2}^{0}(u)=\dot{x}_{1}^{0}(u)$ has poles of order $r=3$ at $u= \pm \pi \mathrm{i}$, and $\ell=2$.

Applying Theorem 1.1 and Corollary 1.2 to the examples (1.5), (1.6) and (1.7), we get the following corollary.

Corollary 1.3 For $\varepsilon \rightarrow 0^{+}, \mu \rightarrow 0$, the following estimates hold:

1. $A=\frac{16}{3} \pi \mu \varepsilon^{p-2} \mathrm{e}^{-\pi / 2 \varepsilon}\left[1+\mathrm{O}\left(\mu \varepsilon^{p-3}, \varepsilon^{4}\right)\right]$, for the pendulum equation (1.5), if $p \geq 3$;

2. $A=\frac{4}{3} \pi \mu \varepsilon^{p-3} \mathrm{e}^{-\pi / 2 \varepsilon}\left[1+\mathrm{O}\left(\mu \varepsilon^{p-3}, \varepsilon^{5}\right)\right]$, for the Duffing equation (1.6), if $p \geq 4$;

3. $A=24 \pi \mu \varepsilon^{p-1} \mathrm{e}^{-\pi / \varepsilon}\left[1+\mathrm{O}\left(\mu \varepsilon^{p}, \varepsilon^{3}\right)\right]$, for the cubic potential equation (1.7), if $p \geq 2$.

\section{Remarks on the hypotheses and the results}

$R 1$ Hypothesis $H 1$ is clear: it requires a separatrix for the unperturbed solution to a saddle point, i.e., $f(0)=0, f^{\prime}(0)>0$, and $\omega^{0}:=\sqrt{f^{\prime}(0)}>0$.

$R 2$ In hypothesis $H 2$, the analyticity of $x_{2}^{0}(u)$ on a complex strip $|\Im u|<a$ is assumed. This is not a restriction for an analytic unperturbed system, since the separatrix behaves regularly for $\Re t \rightarrow \pm \infty$. The real restriction is the assumption that the only singularity of $x_{2}^{0}(u)$ on each component of the boundary of this strip is a pole 
of some order $r \geq 1$, which implies a severe restriction on the behavior of $f\left(x_{1}\right)$ for $x_{1}$ big enough. More precisely, if $r \geq 2$, the equation $f\left(x_{1}^{0}(u)\right)=\ddot{x}_{1}^{0}(u)$ implies that $f\left(x_{1}^{0}(u)\right)$ has a pole of order $r+1$ at $u= \pm a$ i. As $f\left(x_{1}\right)$ is an entire function and $x_{1}^{0}(u)$ has a pole of order $r-1$ at $u= \pm a \mathrm{i}$, it is easy to check that $f\left(x_{1}\right)$ has to be a polynomial of degree 2 or 3 , for $r=3$ or $r=2$, respectively. Analogously, $r=1$ can only take place if $f\left(x_{1}\right)$ is a trigonometric polynomial of degree 1 or 2 , and then $x_{1}^{0}(u) \sim \mathrm{i} k \log (u \mp a \mathrm{i}) \quad(u \rightarrow \pm a \mathrm{i})$, with $k$ equal to 2 or 1 , respectively. All the other values of $r$ and of the degree of the (trigonometric) polynomial $f\left(x_{1}\right)$ give rise to branching points ("poles of fractional order") as singularities of $x_{2}^{0}(u)$.

Actually, our method also applies to $x_{2}^{0}$ with branching points (and even to meromorphic $f$ and $h^{1}$ ), but in this paper we only consider a meromorphic $x_{2}^{0}$ for simplicity and to be able to compute explicitly the Melnikov function. However, it is worth mentioning that in the case of an essential singularity for $x_{2}^{0}$, it seems difficult to control the behavior of the perturbed invariant manifolds, and even it is not clear at all which kind of asymptotics for splitting of separatrices could take place (see [SMH91] for a related discussion).

R3 Through all this paper, the parameter $\ell$ introduced as a consequence of the hypothesis $H 3$ will be called the order of the perturbation on the separatrix or, even more precisely, the order of the perturbation on the singularity of the homoclinic solution. It is worth noting that Theorem 1.1 holds under the assumption $p:=$ power of $\varepsilon \geq \ell=$ order of the perturbation on the separatrix.

By its definition, it is not difficult to observe that $\ell$ satisfies $\ell \geq r-1$. In general, $\ell$ will be the order of the pole of $h^{1}\left(x^{0}(u), \theta\right)$ at $u= \pm a \mathrm{i}$, if there is no cancellation between the different monomials of $h^{1}$, when evaluated on $x^{0}(u)$. An example where these cancellations take place is provided by $h^{1}=h^{0}(x)$. In such case, $h^{0}\left(x^{0}(u)\right)$ is constant (and hence with no pole at all), but for instance the monomial $x_{2}^{2} / 2$ has a pole of order $2 r$. The same happens if $h^{1}$ is functionally dependent on $h^{0}$.

Let us note that in the case $h^{1}(x, \theta)=x_{1} g(\theta)$, system (1.2) is equivalent to the scalar equation $\ddot{x}_{1}=f\left(x_{1}\right)+\mu \varepsilon^{p} g(t / \varepsilon)$, i.e., the perturbation only depends on time. In the trigonometric case, $x_{1}^{0}(u)$ has logarithmic singularities, but the results of Theorem 1.1 are still valid if we take, by convention, $\ell=0$.

R4 Hypothesis H3 is more restrictive than necessary. The hypothesis which is actually used by our method is a little bit more technical and is given in Lemmas 4.2 and 5.1, which simply establish the size of the perturbation $h^{1}(x, \theta)$ and its derivatives for $x$ near $x^{0}(u)$ and $u$ close to $a$ i in terms of the order $\ell$.

Namely, the only important fact is to control the derivatives of $h^{1}$ over the separatrix $x^{0}(u)$ near the singularity $u=a \mathrm{i}$. For instance, if $h^{1}$ has only a monomial 
and $h^{1}\left(x^{0}(u), \theta\right)$ has a pole of order $\ell$, then its derivative with respect to $u$

$$
\begin{aligned}
\frac{\mathrm{d}}{\mathrm{d} u} h^{1}\left(x^{0}(u), \theta\right) & =\left\{h^{0}, h^{1}\right\}\left(x^{0}(u), \theta\right) \\
& =\partial_{1} h^{1}\left(x^{0}(u), \theta\right) x_{2}^{0}(u)+\partial_{2} h^{1}\left(x^{0}(u), \theta\right) \dot{x}_{2}^{0}(u)
\end{aligned}
$$

has a pole of order $\ell+1$, and hence $\partial_{1} h^{1}\left(x^{0}(u), \theta\right)$ has a pole of order $\ell+1-r$ and $\partial_{2} h^{1}\left(x^{0}(u), \theta\right)$ has a pole of order $\ell-r$. The same happens if $h^{1}$ satisfies hypothesis $H 3$ and $\ell$ is the order of the perturbation on the singularity of the homoclinic solution, i.e., $\ell$ is taken as the greatest order of the different monomials of $h^{1}$.

$R 5$ We note that if $h^{1}\left(x^{0}(u), \theta\right)$ has a pole of order $\ell^{\prime} \leq \ell$, then its derivative with respect to $u(1.8)$ has a pole of order $\ell^{\prime}+1$. Hypothesis $H 4$ requires that this pole is realized in the first harmonic with respect the parameter $\theta$, and that $\ell^{\prime}=\ell$, i.e., that there are no cancellations between the different monomials of $h^{1}$, when evaluated on $x^{0}(u)$. It is clear that hypothesis $H 4$ is a generic condition. The case $\ell^{\prime}<\ell$ would require a larger $p$ in order to prove Theorem 1.1 by our methods.

R6 Our measure of the splitting of the separatrices is given by the so-called splitting function $\psi$, which is defined in (2.24), after introducing some suitable "flow-box" canonical coordinates $(S, E)$. In these coordinates, $S$ is a common parameter for both the stable and the unstable manifolds, $E=0$ is the equation of the stable manifold, and $E=\psi(S)$ is the equation for the unstable one. It is important to notice that the splitting function is $2 \pi \varepsilon$-periodic and independent of time, and hence it gives an invariant measure of the distance between the invariant manifolds. In particular, its zeros give rise to homoclinic orbits, and all the splitting quantities are obtained from it. Thus, the area $A$ and the angle $\alpha$ given in Theorem 1.1 are expressed in terms of the integral and the derivative of the splitting function $\psi$. Other related quantities that measure the splitting of separatrices could also be computed. Among them, let us mention the maximum distance $d$ between the invariant curves at the lobe and the Lazutkin homoclinic invariant [GLT91] which is simply $\psi^{\prime}\left(h^{\mathrm{s}}\right)$ in the notation of Proposition 2.7.

$R 7$ Two remarkable recent works related with this paper are [Gel95] and [Gal94].

V. Gelfreich [Gel95] considers a Hamiltonian of the form

$$
H=H_{0}(x, y)+\mu H_{1}(x, y, t / \varepsilon ; \varepsilon, \mu)
$$

and establishes sufficient conditions for the validity of the Melnikov method provided $|\mu|<\mu_{0} \varepsilon^{p}$ for some constant $p$. His method resembles ours, but the constant $p$ relies on the validity of an extension theorem like our Theorem 2.4, which is introduced as an extra hypothesis. Consequently, [Gel95] cannot give explicitly $p$ in concrete examples. 
G. Gallavotti [Gal94] considers the rotator-pendulum model (also called the resonance model) given by a Hamiltonian

$$
\frac{\omega_{0} A}{\varepsilon}+\frac{A^{2}}{2 J}+\frac{I^{2}}{2 J_{0}}+g^{2} J_{0}(\cos \varphi-1)+\mu \sum_{\substack{|n|+|\nu| \leq N \\ \nu \neq 0}} f_{n, \nu} \cos (\alpha \nu+n \varphi)
$$

where $(\varphi, I) \in \mathbf{R}^{2}$ and $(\alpha, A)$ are canonically conjugated variables and $\omega_{0}, J_{0}, J$, $g$ are positive parameters with $J_{0} \leq J \leq \infty$. Under the assumption $|\mu| \leq \varepsilon^{2 q}$, $q>3 N_{0}+5$, where $N_{0}$ is the degree in $\varphi$ of the trigonometric polynomial above, he proves that the splitting distance is given by the Melnikov function. His proof is based on an algorithm derived in [CG94] for the computation of the $\mu$-expansion coefficients of the invariant manifolds and the exponential smallness is obtained explicitly checking cancellation mechanisms, operating to all orders.

We emphasize that his method can be applied to a broader setting, i.e., $n$-degrees of freedom Hamiltonians, and that his condition above on $q$ for $n=2$ reads as $p>6 N_{0}+10=3 \ell+10$ in our notation (or $p>\ell+9$, following some ideas given in the appendix of [Gal94]), whereas in this paper, we only require $p \geq \ell$.

$R 8$ Concerning optimality of $p$, our estimates are valid for $p \geq \ell$, which is the condition required for the Extension Theorem 2.4 in the complex strip (2.16). We believe that this Extension Theorem is not true if $p<\ell$ (this has to do with the fact that the term $\mu \varepsilon^{p} M(s, \varepsilon)$ of the Melnikov method is not small in the complex strip $|\Im s| \leq a-\varepsilon$ for $p<\ell$ ). Of course, we do not claim that $p \geq \ell$ is the optimal lower bound, but it is clear that new methods are needed for lower ranges of $p$. For instance, D. Treschev [Tre97], using a continuous averaging method, proves for an specific trigonometric example with $\ell=2$, that the splitting is given by the Melnikov method for $p>0=\ell-2$. Also in the trigonometric case, G. Gallavotti [Gal94] gives $p>\ell-1$ as a probably optimal lower bound, and recent papers by C. Simó [Sim94] and V. Gelfreich [Gel97], as well as numerical experiments, seem to indicate that the lower bound can be $p>\ell-2$.

The structure of this paper is devoted to give full and comprehensible details of the proof of the Main Theorem 1.1, and more concretely, of the construction of the splitting function. In section 2, all the main ideas are introduced, as well as the main tools: first, the Normal Form Theorem and its Corollaries, which provide good (complex) parameterizations for the local invariant manifolds associated to the periodic orbit of system (1.2), as well as Flow Box Coordinates near the local stable manifold; second, the Extension Theorem, which justifies the prolongation of the unstable manifold until it passes again near the periodic orbit; and third, Propositions 2.6 and 2.7, which allow us to define the splitting function $\psi$, to relate it to the Melnikov function and give, as a direct consequence, the proof of the Main Theorem. To avoid a premature incursion into technicalities, the proofs of these tools, as well as Corollary 1.2, are deferred to successive sections. 
Splitting of separatrices in Hamiltonian systems with $1 \frac{1}{2}$ degrees of freedom

\section{Proof of the Main Theorem}

From now on, hypotheses $H 1-H 3$ and the condition $\gamma=p-\ell \geq 0$ will be implicitly assumed, and $\varepsilon>0$ and $\mu$ will denote small enough independent parameters. To avoid a cumbersome notation, the dependence of all the functions on $\mu, \varepsilon$ is not explicitly written, but is always assumed continuous and bounded.

First of all, the next theorem deals with the local behavior of system (1.2) and states that the Birkhoff normal form is convergent in a neighborhood of the origin.

Theorem 2.1 (Normal Form Theorem) The following properties are satisfied for the system (1.2):

1. There exists a $2 \pi \varepsilon$-periodic orbit near the origin $\gamma_{p}(t / \varepsilon)$, with

$$
\gamma_{p}(\theta)=\left(\gamma_{1}(\theta), \gamma_{2}(\theta)\right)=\mu \varepsilon^{p+1} G(0, \theta)+\mathrm{O}\left(\mu \varepsilon^{p+2}\right)
$$

where $G=\left(G_{1}, G_{2}\right)$ is related to $h^{1}$ through

$$
\partial_{\theta} G(x, \theta)=\left(\partial_{2} h^{1}(x, \theta),-\partial_{1} h^{1}(x, \theta)\right), \quad \int_{0}^{2 \pi} G(x, \theta) \mathrm{d} \theta=0
$$

2. There exists a canonical change of variables

$$
(X, \theta=t / \varepsilon) \longmapsto(x=\Psi(X, \theta), \theta)
$$

with

$$
\Psi(X, \theta)=\Psi(X, \theta, \mu, \varepsilon)=\Psi^{0}(X)+\mu \varepsilon^{p+1} G\left(\Psi^{0}(X), \theta\right)+\mathrm{O}\left(\mu \varepsilon^{p+2}\right),
$$

analytic in $X$ and $2 \pi$-periodic and $\mathcal{C}^{2}$ in $\theta$ for $\left|X_{1}\right|^{2}+\left|X_{2}\right|^{2}<R_{0}^{2}, \theta \in \mathbf{R}$, with $R_{0}$ independent of $\varepsilon, \mu$, such that transforms system (1.2) into its normal form:

$$
\dot{X}_{1}=F^{\prime}\left(X_{1} X_{2}, \mu, \varepsilon\right) X_{1}, \quad \dot{X}_{2}=-F^{\prime}\left(X_{1} X_{2}, \mu, \varepsilon\right) X_{2} .
$$

This system is a Hamiltonian system with associated Hamiltonian

$$
H(X, \theta, \mu, \varepsilon):=F\left(X_{1} X_{2}, \mu, \varepsilon\right)=F^{0}\left(X_{1} X_{2}\right)+\mu \varepsilon^{p+2} F^{1}\left(X_{1} X_{2}, \mu, \varepsilon\right),
$$

with

$$
F(I, \mu, \varepsilon)=\omega I+\mathrm{O}\left(I^{2}\right), \quad F^{\prime}(I, \mu, \varepsilon)=\omega+\mathrm{O}(I), \quad \omega=\omega^{0}+\mathrm{O}\left(\mu \varepsilon^{p+2}\right),
$$

where $F^{\prime}(I, \mu, \varepsilon)$ denotes the derivative of $F$ with respect to its first variable $I$.

3. The change of variables $x=\Psi^{0}(X)$ transforms system (1.1) into its normal form, which is the Hamiltonian system associated to $F^{0}\left(X_{1} X_{2}\right)=\omega^{0} X_{1} X_{2}+\mathrm{O}\left(X_{1}^{2} X_{2}^{2}\right)$. 
From a quantitative point of view, Theorem 2.1 states that: (i) the region of convergence of the normal form is independent of $\varepsilon, \mu$; (ii) the periodic orbit $\gamma_{p}$ and the change of variables $\Psi$ are $\mathrm{O}\left(\mu \varepsilon^{p+1}\right)$-close to the unperturbed ones: 0 and $\Psi^{0}$, respectively; and (iii) the normal form $F$ and the characteristic exponent $\omega$ are $\mathrm{O}\left(\mu \varepsilon^{p+2}\right)$-close to $F^{0}$ and $\omega^{0}$, respectively (this extra $\varepsilon$ is due to the zero mean of $h^{1}$ ). Its proof is based on a parameterized version of a well known theorem due to Moser [Mos56], and is deferred to section 3. More recent proofs, valid for more degrees of freedom, can be found in [CG94, DGJS96, RW97a].

Since system (2.4) can be explicitly solved, Theorem 2.1 supplies us with the basic tools to control the local behavior of the orbits. In particular, good parameterizations $x^{\mathrm{u}}(t, s)$ and $x^{\mathrm{s}}(t, s)$ for the local invariant manifolds, $W_{\mathrm{loc}}^{\mathrm{u}}\left(\gamma_{p}\right)$ and $W_{\mathrm{loc}}^{\mathrm{s}}\left(\gamma_{p}\right)$, are easily found and their properties are summarized in the next corollary.

Corollary 2.2 (Local Invariant Manifolds) There exist parameterizations $x^{\mathrm{u}}(t, s)$, $x^{\mathrm{s}}(t, s)$ of the unstable and stable invariant local manifolds of system (1.2), defined in $D^{\mathrm{u}}, D^{\mathrm{s}}$, respectively, with:

$$
\begin{aligned}
D^{\mathrm{u}} & :=\{(t, s) \in \mathbf{R} \times \mathbf{C}: t+\Re s \leq-T / 2\}, \\
D^{\mathrm{s}} & :=\{(t, s) \in \mathbf{R} \times \mathbf{C}: t+\Re s \geq T / 2\},
\end{aligned}
$$

with $T$ some constant independent of $\mu, \varepsilon$, which satisfy the following properties (* stands for $\mathrm{s}$ and $\mathrm{u})$ :

1. $t \mapsto x^{*}(t, s)$ is a solution of system (1.2) and $s \mapsto x^{*}(t, s)$ is real analytic.

2. $x^{*}(t+2 \pi \varepsilon, s)=x^{*}(t, s+2 \pi \varepsilon)$, and thus the local stable and local unstable curves for the Poincaré map (1.3) are given by $C_{\mathrm{loc}}^{*}=\left\{x^{*}(2 \pi n \varepsilon, s)\right\}$, for any $n \in \mathbf{N}$ such that $(2 \pi n \varepsilon, s) \in D^{*}$.

3. For $\mu=0, x^{*}(t, s)$ coincides with the homoclinic solution $x^{0}(t+s)$, and for $\mu \neq 0$ the following estimate holds:

$$
x^{*}(t, s)=x^{0}(t+s)+\mu \varepsilon^{p+1} G\left(x^{0}(t+s), t / \varepsilon\right)+\mathrm{O}\left(\mu \varepsilon^{p+2}\right) .
$$

4. Near the periodic orbit $\gamma_{p}$, the following asymptotics are satisfied:

$$
\begin{aligned}
x^{\mathrm{u}}(t, s) & =\gamma_{p}(t / \varepsilon)+x^{0}(t+s)+\mathrm{O}\left(\mu \varepsilon^{p+1} \mathrm{e}^{\omega(t+s)}\right)+\mathrm{O}\left(\mu \varepsilon^{p+2} \mathrm{e}^{(t+s) / 2}\right), \\
x^{\mathrm{s}}(t, s) & =\gamma_{p}(t / \varepsilon)+x^{0}(t+s)+\mathrm{O}\left(\mu \varepsilon^{p+1} \mathrm{e}^{-\omega(t+s)}\right)+\mathrm{O}\left(\mu \varepsilon^{p+2} \mathrm{e}^{-(t+s) / 2}\right),
\end{aligned}
$$

$\omega$ being the positive constant defined in formula (2.6) as the characteristic exponent of the periodic orbit $\gamma_{p}$. 
The parameterizations $x^{\mathrm{u}}(t, s), x^{\mathrm{s}}(t, s)$, can be considered "natural" [DR96], since they are formed by solutions of system (1.2) in the real variable $t$, and the action of the Poincaré map is simply a shift of amount $2 \pi \varepsilon$ in the complex variable $s$. It is worth mentioning that they are not uniquely determined, because a new parameterization $\tilde{x}^{*}(t, S)=x^{*}(t, S+\phi(S))$ (i.e., a change of parameter $\left.s=S+\phi(S)\right)$, for a $2 \pi \varepsilon$-periodic function $\phi$ of size $\mathrm{O}\left(\mu \varepsilon^{p+1}\right)$, does not change the properties above, but it may change the domain of analyticity of $S \mapsto \tilde{x}^{*}(t, S)$. We will use this freedom of choice later on.

Another consequence of the explicit solution of system (2.4) is that performing the change of variables $S=-\log X_{2} / F^{\prime}\left(X_{1} X_{2}\right), E=F\left(X_{1} X_{2}\right)$, and composing it with the change of variables (2.2) to normal form, one obtains the existence of local flow box coordinates outside of the local unstable invariant manifold $W_{\mathrm{loc}}^{\mathrm{u}}\left(\gamma_{p}\right)$.

Corollary 2.3 (Flow Box Theorem) There exists a canonical change of variables

$$
(x, \theta=t / \varepsilon) \in \mathcal{U} \longmapsto(S, E, \theta)=(\mathcal{S}(x, \theta), \mathcal{E}(x, \theta), \theta) \in \mathcal{V},
$$

analytic in $x, 2 \pi$-periodic and $\mathcal{C}^{2}$ in $\theta$ on $\mathcal{U}=\left\{(x, \theta) \in \mathbf{C}^{2} \times \mathbf{R}:\left\|x-\gamma_{p}(\theta)\right\|<\right.$ $\left.r_{0}^{2}\right\} \backslash W_{\mathrm{loc}}^{\mathrm{u}}\left(\gamma_{p}\right)$, with $r_{0}$ independent of $\mu, \varepsilon$, such that transforms system (1.2) in a flow box system

$$
\dot{S}=1, \quad \dot{E}=0
$$

and satisfies:

1.

$$
\mathcal{S}(x, \theta)=\mathcal{S}^{0}(x)+\mathrm{O}\left(\mu \varepsilon^{p+1}\right), \quad \mathcal{E}(x, \theta)=\mathcal{E}^{0}(x)+\mathrm{O}\left(\mu \varepsilon^{p+1}\right),
$$

where $x \mapsto\left(\mathcal{S}^{0}(x), \mathcal{E}^{0}(x)=h^{0}(x)\right)$ is the corresponding change for system (1.1).

2. Denoting $(S, E, \theta) \in \mathcal{V} \longmapsto(\mathcal{X}(S, E, \theta), \theta) \in \mathcal{U}$ the inverse change to (2.11), the following estimate holds

$$
\mathcal{X}(S, E, \theta)=\mathcal{X}^{0}(S, E)+\mathrm{O}\left(\mu \varepsilon^{p+1}\right),
$$

where $x=\mathcal{X}^{0}(S, E)$ is the inverse change to $x \mapsto(S, E)=\left(\mathcal{S}^{0}(x), \mathcal{E}^{0}(x)\right)$.

3. Along the local stable manifold $x^{\mathrm{s}}(t, s)$, the flow-box functions (2.11) satisfy

$$
\mathcal{S}\left(x^{\mathrm{s}}(t, s), t / \varepsilon\right)=t+s, \quad \mathcal{E}\left(x^{\mathrm{s}}(t, s), t / \varepsilon\right)=0
$$

Up to now, the parameterization $x^{\mathrm{u}}(t, s)$ of the unstable manifold has been only defined for $(t, s) \in D^{\mathrm{u}}$ given by (2.7). To extend it for other values of $(t, s)$, we would like to use an analog of estimate $(2.9)$, which relates $x^{\mathrm{u}}(t, s)$ to the unperturbed separatrix $x^{0}(t+s)$. However, $x^{0}(u)$ has a singularity in the complex field at $u= \pm a \mathrm{i}$; more precisely, since $x_{2}^{0}(u)=\dot{x}_{1}^{0}(u)$ has a pole of order $r$ at these points, it has the form

$$
x_{2}^{0}(u)=\frac{\mp \mathrm{Ci}}{(u \mp a \mathrm{i})^{r}}(1+\mathrm{O}(u \mp a \mathrm{i})),
$$


with $\mathrm{C}$ a non-zero constant, for $u$ near $\pm a \mathrm{i}$. This means that we will not be able to control the growth of the parameterization for $t+s= \pm a$ i, i.e., for $\Im s= \pm a$, since the time $t$ is always assumed to be real. We will restrict ourselves, as in [DS92], to a complex strip $\mathcal{D}_{\varepsilon}^{\mathrm{u}}$ of imaginary width equal to $a-\varepsilon$ :

$$
\mathcal{D}_{\varepsilon}^{\mathrm{u}}:=\{(t, s) \in \mathbf{R} \times \mathbf{C}: \quad|\Im s| \leq a-\varepsilon, \quad|t+\Re s| \leq T\} .
$$

The following Extension Theorem ensures us that the parameterization $x^{\mathrm{u}}(t, s)$ of the unstable invariant manifold $W^{\mathrm{u}}\left(\gamma_{p}\right)$ is still defined and close enough to the unperturbed separatrix. We state it first, for the sake of generality, for arbitrary solutions $x(t, s)$ of $(1.2)$.

Theorem 2.4 (Extension Theorem) Let $x^{0}(t+s)$ be the unperturbed separatrix of system (1.1), and $x(t, s)$ a family of solutions of (1.2) such that

$$
x\left(t_{0}, s\right)-x^{0}\left(t_{0}+s\right)-\mu \varepsilon^{p+1} G\left(x^{0}\left(t_{0}+s\right), t_{0} / \varepsilon\right)=\mathrm{O}\left(\mu \varepsilon^{p+2}\right),
$$

where $s \in \mathbf{C},|\Im s| \leq a-\varepsilon$, and $t_{0}+\Re s=-T$.

Then, if $\gamma=p-\ell \geq 0, x(t, s)$ is defined on $\mathcal{D}_{\varepsilon}^{\mathrm{u}}$ given in (2.16) and satisfies there:

$$
x(t, s)-x^{0}(t+s)=\mathrm{O}\left(\mu \varepsilon^{\gamma}\right) .
$$

The proof of this theorem involves several technicalities, such as a good choice of the solutions of the variational equations associated to the separatrix and the partition of the strip $\mathcal{D}_{\varepsilon}^{\mathrm{u}}$ in different regions. Apart from this, it relies on straightforward estimates, following the same arguments as those in [DS92, DGJS96, RW97b], and is deferred to section 4.

By estimate (2.9), it is clear that the parameterization $x^{\mathrm{u}}(t, s)$ of $W_{\text {loc }}^{\mathrm{u}}\left(\gamma_{p}\right)$ satisfies the hypotheses of the Extension Theorem for $t_{0}=-\Re s-T$, and consequently the following estimate holds

$$
x^{\mathrm{u}}(t, s)-x^{0}(t+s)=\mathrm{O}\left(\mu \varepsilon^{\gamma}\right), \quad(t, s) \in \mathcal{D}_{\varepsilon}^{\mathrm{u}} .
$$

Remark 2.5 It has to be noticed here that for fixed $T$ and $s \in \mathbf{R}$, this Extension Theorem becomes a well-known result and estimate (2.18) is of the same order as the one at initial condition (2.17), i.e.,

$$
x^{\mathrm{u}}(t, s)-x^{0}(t+s)=\mu \varepsilon^{p+1} G\left(x^{0}(t+s), t / \varepsilon\right)+\mathrm{O}\left(\mu \varepsilon^{p+2}\right),
$$

for $-T \leq t+s \leq T$, and $t, s \in \mathbf{R}$.

By hypothesis $H 1$, and more precisely by estimate $(2.10), x^{0}(t+s)$ arrives and stays at the open set $\mathcal{U}$ for $t+\Re s \geq T / 2$. By estimate (2.18), the same happens to $x^{\mathrm{u}}(t, s)$ for 
$T / 2 \leq t+\Re s \leq T$, and $|\Im s| \leq a-\varepsilon$. On $\mathcal{U}$, the flow-box functions (2.11) are defined, and therefore can be evaluated on the unstable manifold giving rise to two functions:

$$
\mathcal{S}^{\mathrm{u}}(s):=\mathcal{S}\left(x^{\mathrm{u}}(t, s), t / \varepsilon\right)-t, \quad \mathcal{E}^{\mathrm{u}}(s):=\mathcal{E}\left(x^{\mathrm{u}}(t, s), t / \varepsilon\right),
$$

defined for $|\Im s| \leq a-\varepsilon$. In equation (2.20), the time $t$ on the right terms has been chosen in $T / 2 \leq t+\Re s \leq T$, but $\mathcal{S}^{\mathrm{u}}$ and $\mathcal{E}^{\mathrm{u}}$ do not depend on $t$ since, by Corollary 2.3, $\mathcal{S}(x, t / \varepsilon)-t$ and $\mathcal{E}(x, t / \varepsilon)$ are real analytic first integrals of system (1.2). Besides, by properties (1) and (2) of Corollary 2.2, it turns out that $\mathcal{S}^{\mathrm{u}}(s)-s$ and $\mathcal{E}^{\mathrm{u}}(s)$ are analytic for $|\Im s| \leq a-\varepsilon$ and $2 \pi \varepsilon$-periodic in $s$.

The next Proposition asserts that $\mathcal{E}^{\mathrm{u}}(s)$ is well-approximated by the Melnikov function for $|\Im s| \leq a-\varepsilon$, and as a consequence, that the difference between $\mathcal{E}^{\mathrm{u}}(s)-\mathcal{E}_{0}^{\mathrm{u}}$ and $\mu \varepsilon^{p} M(s, \varepsilon)$ is $\mathrm{O}\left(\mathrm{e}^{-a / \varepsilon}\right)$ for real $s$, where $\mathcal{E}_{0}^{\mathrm{u}}$ is the zero order Fourier coefficient of $\mathcal{E}^{\mathrm{u}}$.

Proposition 2.6 For $\gamma=p-\ell \geq 0, \mathcal{S}^{\mathrm{u}}$ and $\mathcal{E}^{\mathrm{u}}$ satisfy the following estimates:

1. For $s \in \mathbf{C}$ such that $|\Im s| \leq a-\varepsilon$,

$$
\mathcal{E}^{\mathrm{u}}(s)=\mu \varepsilon^{p} M(s, \varepsilon)+\mathrm{O}\left(\mu^{2} \varepsilon^{2 \gamma+r-1}, \mu \varepsilon^{p+1}\right) .
$$

2. For $s \in \mathbf{R}$, and $\mathcal{E}_{0}^{\mathrm{u}}=\frac{1}{2 \pi \varepsilon} \int_{0}^{2 \pi \varepsilon} \mathcal{E}^{\mathrm{u}}(s) \mathrm{d} s$,

$$
\mathcal{E}^{\mathrm{u}}(s)-\mathcal{E}_{0}^{\mathrm{u}}=\mu \varepsilon^{p} M(s, \varepsilon)+\mathrm{O}\left(\mu^{2} \varepsilon^{2 \gamma+r-1}, \mu \varepsilon^{p+1}\right) \mathrm{e}^{-a / \varepsilon} .
$$

3. For $s \in \mathbf{R}, S=\mathcal{S}^{\mathrm{u}}(s)$ is real analytic and invertible, and its inverse $s=s^{\mathrm{u}}(S)$ satisfies that $s^{\mathrm{u}}(S)-S$ is $\mathrm{O}\left(\mu \varepsilon^{p+1}\right)$ and $2 \pi \varepsilon$-periodic in $S$.

From Corollary 2.3, and in particular from equations (2.15), it follows that the local stable manifold $x^{\mathrm{s}}(t, s)$ has a very simple expression in the $(S, E)$ coordinates:

$$
(S, E)=\left(\mathcal{S}\left(x^{\mathrm{s}}(t, s), t / \varepsilon\right), \mathcal{E}\left(x^{\mathrm{s}}(t, s), t / \varepsilon\right)\right)=(t+s, 0),
$$

i.e., $E=0$. Using equations $(2.20)$, the arriving unstable manifold $x^{\mathrm{u}}(t, s)$ has in these coordinates the expression

$$
(S, E)=\left(\mathcal{S}\left(x^{\mathrm{u}}(t, s), t / \varepsilon\right), \mathcal{E}\left(x^{\mathrm{u}}(t, s), t / \varepsilon\right)\right)=\left(t+\mathcal{S}^{\mathrm{u}}(s), \mathcal{E}^{\mathrm{u}}(s)\right)
$$

and, in particular, the unstable curve $C^{\mathrm{u}}$ of the Poincaré map $P$ defined in (1.3), is given by $(S, E)=\left(2 \pi n \varepsilon+\mathcal{S}^{\mathrm{u}}(s), \mathcal{E}^{\mathrm{u}}(s)\right)$, using property (2) of Corollary 2.2.

Therefore, it is very natural to introduce the splitting function $\psi$ given implicitly by $\psi\left(2 \pi n \varepsilon+\mathcal{S}^{\mathrm{u}}(s)\right)=\mathcal{E}^{\mathrm{u}}(s)$, or simply by $\psi\left(\mathcal{S}^{\mathrm{u}}(s)\right)=\mathcal{E}^{\mathrm{u}}(s)$, using that $\mathcal{S}^{\mathrm{u}}(s)-s$ and $\mathcal{E}^{\mathrm{u}}(s)$ are $2 \pi \varepsilon$-periodic in $s$. By Proposition 2.6, $S=\mathcal{S}^{\mathrm{u}}(s)$ can be inverted for real values of $s$, giving rise to the inverse function $s=s^{\mathrm{u}}(S)$. Consequently, $\psi(S)$ is explicitly defined, for real values of $S$, as

$$
\psi(S)=\mathcal{E}^{\mathrm{u}}\left(s^{\mathrm{u}}(S)\right)
$$


Since $s^{\mathrm{u}}(S)-S$ is $\mathrm{O}\left(\mu \varepsilon^{p+1}\right)$ and $2 \pi \varepsilon$-periodic in $s$, we can introduce a new natural parameterization for the unstable invariant manifold (see the comments following Corollary 2.2)

$$
\tilde{x}^{\mathrm{u}}(t, S)=x^{\mathrm{u}}\left(t, s^{\mathrm{u}}(S)\right)
$$

and by (2.15) we can simply write for the stable invariant manifold

$$
\tilde{x}^{\mathrm{s}}(t, S)=x^{\mathrm{s}}(t, S)
$$

in such a way that $\psi(S)$ can be also written as

$$
\begin{aligned}
\psi(S) & =\mathcal{E}\left(x^{\mathrm{u}}\left(t, s^{\mathrm{u}}(S)\right), t / \varepsilon\right) \\
& =\mathcal{E}\left(x^{\mathrm{u}}\left(t, s^{\mathrm{u}}(S)\right), t / \varepsilon\right)-\mathcal{E}\left(x^{\mathrm{s}}(t, S), t / \varepsilon\right) \\
& =\mathcal{E}\left(\tilde{x}^{\mathrm{u}}(t, S), t / \varepsilon\right)-\mathcal{E}\left(\tilde{x}^{\mathrm{s}}(t, S), t / \varepsilon\right) \\
& =\mathcal{E}\left(\tilde{x}^{\mathrm{u}}(t, S), t / \varepsilon\right)
\end{aligned}
$$

It only remains to confirm that $\psi$ measures the splitting, and this is done in the next Proposition.

Proposition 2.7 The function $\psi$ is a $2 \pi \varepsilon$-periodic, real analytic function that satisfies the following properties.

1. There exists $h^{\mathrm{u}} \in \mathbf{R}$ such that $x^{\mathrm{u}}\left(t, h^{\mathrm{u}}\right)=x^{\mathrm{s}}\left(t, h^{\mathrm{s}}\right)$ (giving an homoclinic connection), with $h^{\mathrm{s}}=\mathcal{S}^{\mathrm{u}}\left(h^{\mathrm{u}}\right)$. Consequently, $\psi\left(h_{n}\right)=0$, for $h_{n}=h^{\mathrm{s}}+2 \pi \varepsilon n, n \in \mathbf{N}$. Moreover, $\psi^{\prime}\left(h_{n}\right)$ is independent of $n$, and

$$
\psi^{\prime}\left(h_{n}\right)=\frac{\partial x^{\mathrm{s}}}{\partial S}\left(t, h_{n}\right) \wedge \frac{\partial \tilde{x}^{\mathrm{u}}}{\partial S}\left(t, h_{n}\right)=\left\|\frac{\partial x^{\mathrm{s}}}{\partial S}\left(t, h_{n}\right)\right\| \cdot\left\|\frac{\partial \tilde{x}^{\mathrm{u}}}{\partial S}\left(t, h_{n}\right)\right\| \sin \alpha\left(t, h_{n}\right),
$$

where $\wedge$ denotes the exterior product on $\mathbf{R}^{2}$, and $\alpha\left(t, h_{n}\right)$ is the angle between $x^{\mathrm{u}}\left(t, h^{\mathrm{u}}+2 \pi \varepsilon n\right)=\tilde{x}^{\mathrm{u}}\left(t, h_{n}\right)$ and $x^{\mathrm{s}}\left(t, h_{n}\right)$.

2. The area of the lobe between the invariant curves is given by $A=\left|\int_{h_{n}}^{\bar{h}_{n}} \psi(S) \mathrm{d} S\right|$, where $h_{n}$ and $\bar{h}_{n}$ are the two consecutive zeros of $\psi(S)$ closest to zero.

3. $\psi_{0}=\int_{h_{n}}^{h_{n}+2 \pi \varepsilon} \psi(S) \mathrm{d} S=0$.

4. $\psi(S)$ satisfies for $S \in \mathbf{R}$ the estimate

$$
\psi(S)=\mu \varepsilon^{p} M(S, \varepsilon)+\mathrm{O}\left(\mu^{2} \varepsilon^{2 \gamma+r-1}, \mu \varepsilon^{p+1}\right) \mathrm{e}^{-a / \varepsilon} .
$$

Now all the statements of Theorem 1.1 follow from Proposition (2.7) and specially from the approximations given in (2.25). 


\section{Proof of the Normal Form Theorem and its Cor- ollaries}

\section{Proof of Theorem 2.1}

First of all, it is better to make the change of time $\theta=t / \varepsilon$ to system (1.2). Denoting by ' the derivative with respect to $\theta$, we obtain

$$
\begin{aligned}
& x_{1}^{\prime}=\varepsilon x_{2}+\mu \varepsilon^{p+1} \partial_{2} h^{1}(x, \theta), \\
& x_{2}^{\prime}=\varepsilon f\left(x_{1}\right)-\mu \varepsilon^{p+1} \partial_{1} h^{1}(x, \theta),
\end{aligned}
$$

which is a Hamiltonian system with Hamiltonian

$$
\varepsilon h(x, \theta, \mu, \varepsilon)=\varepsilon h^{0}(x)+\mu \varepsilon^{p+1} h^{1}(x, \theta) .
$$

System (3.1) will be put into its normal form with the aid of several lemmas. As a first step, we deal with its averaged system.

Lemma 3.1 There exists a canonical change

$$
\bar{x} \longmapsto x=\Pi(\bar{x}, \theta, \mu, \varepsilon)=\bar{x}+\mu \varepsilon^{p+1} G(\bar{x}, \theta)+\mathrm{O}\left(\mu \varepsilon^{p+2}\right),
$$

$2 \pi$-periodic and $\mathcal{C}^{2}$ in $\theta$, and analytic in $\bar{x}$, with $G$ defined in (2.1), such that it transforms system (3.1) into a Hamiltonian system with Hamiltonian $\varepsilon \mathcal{H}$, where

$$
\mathcal{H}(\bar{x}, \theta, \mu, \varepsilon)=h^{0}(\bar{x})+\mu \varepsilon^{p+2} \mathcal{R}(\bar{x}, \theta, \mu, \varepsilon),
$$

$\mathcal{R}$ being analytic with respect to $\bar{x}$, and $2 \pi$-periodic and $\mathcal{C}^{1}$ in $\theta$.

Proof. We only have to check that the generating function

$$
S\left(x_{1}, \bar{x}_{2}, \theta, \mu, \varepsilon\right)=x_{1} \bar{x}_{2}+\mu \varepsilon^{p+1} S^{1}\left(x_{1}, \bar{x}_{2}, \theta\right)+\mu \varepsilon^{p+2} S^{2}\left(x_{1}, \bar{x}_{2}, \theta\right)
$$

defined as the solution of the equations:

$$
\begin{aligned}
& \partial_{\theta} S^{1}\left(x_{1}, \bar{x}_{2}, \theta\right)=-h^{1}\left(x_{1}, \bar{x}_{2}, \theta\right), \quad \int_{0}^{2 \pi} S^{1}\left(x_{1}, \bar{x}_{2}, \theta\right) \mathrm{d} \theta=0 \\
& \partial_{\theta} S^{2}\left(x_{1}, \bar{x}_{2}, \theta\right)=V^{\prime}\left(x_{1}\right) \partial_{2} S^{1}\left(x_{1}, \bar{x}_{2}, \theta\right)-\bar{x}_{2} \partial_{1} S^{1}\left(x_{1}, \bar{x}_{2}, \theta\right)
\end{aligned}
$$

provides implicitly, through $\bar{x}_{1}=\partial_{2} S\left(x_{1}, \bar{x}_{2}, \theta, \mu, \varepsilon\right), x_{2}=\partial_{1} S\left(x_{1}, \bar{x}_{2}, \theta, \mu, \varepsilon\right)$, a canonical change $\Pi$ satisfying (3.2) and (3.3). Along this proof, $\partial_{2}$ denotes $\partial / \partial \bar{x}_{2}$.

We first note that by hypothesis $H 3, h^{1}$ has zero mean. Consequently, there exist functions $S^{1}, S^{2}, 2 \pi$-periodic in $\theta$, that are solutions of the equations 3.4 , giving rise to a canonical change $\Pi$ of the form (3.2). Under this change, the new Hamiltonian $\varepsilon \mathcal{H}$ satisfies

$$
\varepsilon \mathcal{H}\left(\partial_{2} S, \bar{x}_{2}, \theta, \mu, \varepsilon\right)=\varepsilon h\left(x_{1}, \partial_{1} S, \theta, \mu, \varepsilon\right)+\partial_{\theta} S,
$$


for $S=S\left(x_{1}, \bar{x}_{2}, \theta, \mu, \varepsilon\right)$, and the right side of this equality can be expanded, using the definitions of $S^{1}, S^{2}$, to

$$
\begin{aligned}
\varepsilon\left(\bar{x}_{2}^{2} / 2+\right. & \left.V\left(x_{1}\right)\right)+\mu \varepsilon^{p+1}\left(h^{1}\left(x_{1}, \bar{x}_{2}, \theta\right)+\partial_{\theta} S^{1}\left(x_{1}, \bar{x}_{2}, \theta\right)\right) \\
& +\mu \varepsilon^{p+2}\left(\bar{x}_{2} \partial_{1} S^{1}\left(x_{1}, \bar{x}_{2}, \theta\right)+\partial_{\theta} S^{2}\left(x_{1}, \bar{x}_{2}, \theta\right)\right)+\mathcal{R}_{1}\left(x_{1}, \bar{x}_{2}, \theta, \mu, \varepsilon\right), \\
= & \varepsilon\left(\bar{x}_{2}^{2} / 2+V\left(x_{1}\right)\right)+\mu \varepsilon^{p+2} V^{\prime}\left(x_{1}\right) \partial_{2} S^{1}\left(x_{1}, \bar{x}_{2}, \theta\right)+\mathcal{R}_{1}\left(x_{1}, \bar{x}_{2}, \theta, \mu, \varepsilon\right),
\end{aligned}
$$

with $\mathcal{R}_{1}\left(x_{1}, \bar{x}_{2}, \theta, \mu, \varepsilon\right)=\mu^{2} \varepsilon^{2 p+2} \partial_{2} h^{1}\left(x_{1}, \bar{x}_{2}, \theta\right) \partial_{1} S^{1}\left(x_{1}, \bar{x}_{2}\right)+\mathrm{O}\left(\mu \varepsilon^{p+3}\right)$. Now, inverting

$$
\bar{x}_{1}=\partial_{2} S\left(x_{1}, \bar{x}_{2}, \theta\right)=x_{1}+\mu \varepsilon^{p+1} \partial_{2} S^{1}\left(x_{1}, \bar{x}_{2}, \theta\right)+\mu \varepsilon^{p+2} \partial_{2} S^{2}\left(x_{1}, \bar{x}_{2}, \theta\right)
$$

we get an expression for $x_{1}$

$$
x_{1}=\bar{x}_{1}-\mu \varepsilon^{p+1} \partial_{2} S^{1}\left(\bar{x}_{1}, \bar{x}_{2}, \theta\right)+\mathrm{O}\left(\mu \varepsilon^{p+2}\right),
$$

which substituted together with (3.5) in the equation for $\varepsilon \mathcal{H}$, reads as

$$
\varepsilon \mathcal{H}(\bar{x}, \theta, \mu, \varepsilon)=\varepsilon\left(\bar{x}_{2}^{2} / 2+V\left(\bar{x}_{1}\right)\right)+\mathcal{R}_{2}\left(\bar{x}_{1}, \bar{x}_{2}, \theta, \mu, \varepsilon\right),
$$

with $\mathcal{R}_{2}=\mu^{2} \varepsilon^{2 p+2} \partial_{2} h^{1} \partial_{1} S^{1}+\mathrm{O}\left(\mu \varepsilon^{p+3}\right)$.

It only remains to see that $\mu^{2} \varepsilon^{2 p+2} \partial_{2} h^{1} \partial_{1} S^{1}=\mathrm{O}\left(\mu \varepsilon^{p+3}\right)$. We distinguish two cases: $\ell \geq 1$ or $\ell=0$. If $\ell \geq 1$, then $\gamma=p-\ell \geq 0$ implies $p \geq \ell \geq 1$, and $\mu^{2} \varepsilon^{2 p+2} \partial_{2} h^{1} \partial_{1} S^{1}=$ $\mathrm{O}\left(\mu^{2} \varepsilon^{p+3}\right)=\mathrm{O}\left(\mu \varepsilon^{p+3}\right)$. If $\ell=0$, this means that we are dealing with the special case $h^{1}(x, \theta)=x_{1} g(\theta)$ of the trigonometric case of hypothesis H3, (see remark R3), i.e., $h^{1}$ does not depend on $x_{2}$ and then $\mu^{2} \varepsilon^{2 p+2} \partial_{2} h^{1} \partial_{1} S^{1}=0$.

Remark 3.2 It could seem a good idea to average the original Hamiltonian $h$ in order to increase the order of the perturbation term (in fact it was $\mathrm{O}\left(\mu \varepsilon^{p}\right)$, and after the averaging it becomes $\mathrm{O}\left(\mu \varepsilon^{p+2}\right)$ ). The question now is when to stop this process. The Hamiltonian we have obtained after two steps of averaging has the same integrable part, but the perturbation term $\mathcal{R}$ does not have zero mean. If we average again, the new Hamiltonian will have another integrable part with a different separatrix that will depend on $\varepsilon$. This separatrix can have a different kind of singularity and hypotheses H1H3 can change drastically.

The averaged Hamiltonian $\mathcal{H}$ has the same integrable part $h^{0}$ as $h$, but the perturbation term is now $\mathrm{O}\left(\mu \varepsilon^{p+2}\right)$. We will see that this order of perturbation is preserved when one considers the Birkhoff normal form of $\mathcal{H}$. First of all let us look for the periodic orbit of $\mathcal{H}$.

Lemma 3.3 The Hamiltonian system with Hamiltonian $\varepsilon \mathcal{H}$ has a hyperbolic $2 \pi$-periodic orbit $\bar{\gamma}_{p}(\theta)=\left(\bar{\gamma}_{1}(\theta), \bar{\gamma}_{2}(\theta)\right)$, which is $\mathrm{O}\left(\mu \varepsilon^{p+2}\right)$. 
Proof. In order to prove this lemma, we only need to consider the Poincaré map associated to the $2 \pi$-periodic Hamiltonian system associated to $\varepsilon \mathcal{H}$. For $\mu \varepsilon^{p+2}=0$ the origin is a hyperbolic fixed point that is preserved for $\mu \varepsilon^{p+2}$ small enough. This new fixed point gives rise to the hyperbolic $2 \pi$-periodic orbit $\bar{\gamma}_{p}$. For an explicit construction of $\bar{\gamma}_{p}$ see [DS92, page 439].

In order to study the local behavior of the orbits near $\bar{\gamma}_{p}$ we perform the canonical change of variables

$$
y=\bar{x}-\bar{\gamma}_{p}(\theta),
$$

that sends the periodic orbit to the origin, and we obtain again a Hamiltonian system with Hamiltonian $\varepsilon k$, with

$$
k(y, \theta, \mu, \varepsilon)=h^{0}(y)+\mu \varepsilon^{p+2} \mathcal{K}(y, \theta, \mu, \varepsilon)=\frac{y_{2}^{2}}{2}-\frac{\left(\omega^{0} y_{1}\right)^{2}}{2}+\mathrm{O}\left(y^{3}, \mu \varepsilon^{p+2}\right),
$$

which is transformed into its normal form up to order two in the following lemma.

Lemma 3.4 There exists a canonical change of variables

$$
Y \longmapsto y=A(\theta, \mu, \varepsilon) Y
$$

linear in $Y$ and $2 \pi$-periodic and $\mathcal{C}^{2}$ in $\theta$, with $A=A^{0}+\mathrm{O}\left(\mu \varepsilon^{p+2}\right)$, where

$$
A^{0}=\frac{1}{2}\left(\begin{array}{cc}
2 / c & -c / \omega^{0} \\
2 \omega^{0} / c & c
\end{array}\right)
$$

$c \neq 0$ being an arbitrary constant, such that it transforms the Hamiltonian system associated to $\varepsilon k$ into a Hamiltonian system whose Hamiltonian $\varepsilon K$ is in normal form up to order 2:

$$
K(Y, \theta, \mu, \varepsilon)=K^{0}(Y)+\mu \varepsilon^{p+2} K^{1}(Y, \theta, \mu, \varepsilon)=\omega Y_{1} Y_{2}+\mathrm{O}\left(Y^{3}\right),
$$

with $\omega=\omega^{0}+\mathrm{O}\left(\mu \varepsilon^{p+2}\right)$.

Proof. It follows directly from Floquet theory applied to the linear part of the Hamiltonian system associated to $\varepsilon k$. In fact, the unperturbed change of variables $y=A^{0} Y$, with $A^{0}$ given in (3.8), transforms the unperturbed Hamiltonian $h^{0}(y)$ in its normal form up to order two, i.e.,

$$
h^{0}(y)=K^{0}(Y)=\omega^{0} Y_{1} Y_{2}+\mathrm{O}\left(Y^{3}\right) .
$$

The Hamiltonian $K$ is now ready to be subject to the nonlinear normal form, which in this case is convergent in a neighborhood of the origin $Y=0$ with a radius that does not depend on $\mu, \varepsilon$. 
Proposition 3.5 There exist $R_{0}>0$, independent of $\mu, \varepsilon$, and a canonical change of variables:

$$
Y=\Phi(X, \theta, \mu, \varepsilon)=\Phi^{0}(X)+\mu \varepsilon^{p+2} \Phi^{1}(X, \theta, \mu, \varepsilon),
$$

analytic with respect to $X$ for $\|X\|^{2}<R_{0}^{2}$ and $2 \pi$-periodic and $\mathcal{C}^{2}$ in $\theta$, and that satisfies

$$
\Phi(X, \theta, \mu, \varepsilon)=X+\mathrm{O}\left(X^{2}\right), \quad \Phi^{0}(X)=X+\mathrm{O}\left(X^{2}\right),
$$

such that transforms the Hamiltonian system associated to $\varepsilon K$ into the Hamiltonian system generated by a Hamiltonian $\varepsilon H$ in normal form

$$
H(X, \theta, \mu, \varepsilon)=F\left(X_{1} X_{2}, \mu, \varepsilon\right)=F^{0}\left(X_{1} X_{2}\right)+\mu \varepsilon^{p+2} F^{1}\left(X_{1} X_{2}, \mu, \varepsilon\right),
$$

with $F(I)=\omega I+\mathrm{O}\left(I^{2}\right)$.

Proof. For fixed $\varepsilon$ and $\mu$, the existence of such a canonical change of variables is a very well known result, due to Moser [Mos56]. It is not difficult to check in that proof that the dependence of the Hamiltonian on $\varepsilon$ and $\mu$ is smooth and in particular that the radius of convergence of the normal form can be bounded from below by a constant independent of both parameters. A complete proof for a fast quasiperiodic forced pendulum can be found in [DGJS96].

Notice also that the unperturbed change $\Phi^{0}(X)$ transforms the system associated to $K^{0}(Y)$ into its normal form, which is the system associated to $F^{0}\left(X_{1} X_{2}\right)$.

Now the proof of Theorem 2.1 is clear if we compose all the changes given by (3.2), (3.6), (3.7) and (3.9), and the change of time $\theta=t / \varepsilon$.

\section{Proof of Corollary 2.2}

By Theorem 2.1, the canonical change of variables (2.2) transforms the original system (1.2) into its normal form given by system (2.4). Then all the results we can get near the origin of system (2.4) can be transported to (1.2) using this change. More specifically we will work in $W=\left\{X \in \mathbf{C}^{2}:\|X\|^{2} \leq R_{0}^{2}\right\}$, and then we will obtain results in $\left\{(x, \theta) \in \mathbf{C}^{2} \times \mathbf{R}:\left\|x-\gamma_{p}(\theta)\right\|<r_{0}^{2}\right\}$, with $r_{0}$ independent of $\mu, \varepsilon$,

For the proof of this corollary we only need to consider the branch of the stable manifold of system $(2.4)$ given by $\left(0, \mathrm{e}^{-\omega t} X_{2}^{\mathrm{s}}\right)$, where $X_{2}^{\mathrm{s}}>0$. Introducing $s+c^{\mathrm{s}}=$ $-\frac{1}{\omega} \log X_{2}^{\mathrm{s}}$, it can be parameterized by

$$
X^{\mathrm{s}}(t, s)=\left(0, \mathrm{e}^{-\omega\left(t+s+c^{\mathrm{s}}\right)}\right) .
$$

The unstable manifold can be parameterized analogously by

$$
X^{\mathrm{u}}(t, s)=\left(\mathrm{e}^{\omega\left(t+s+c^{\mathrm{u}}\right)}, 0\right) .
$$

In fact all the parameters that appear in these formulae are not independent but we will fix them later on. In order to transport all these parameterizations to the original 
system (1.2), we only need to compose them with the change of variables (2.2), and thus we obtain

$$
\begin{aligned}
x^{\mathrm{u}}(t, s) & =\Psi\left(\mathrm{e}^{\omega\left(t+s+c^{\mathrm{u}}\right)}, 0, t / \varepsilon\right), \quad \Re\left(t+s+c^{\mathrm{u}}\right) \leq-T^{0}, \\
x^{\mathrm{s}}(t, s) & =\Psi\left(0, \mathrm{e}^{-\omega\left(t+s+c^{\mathrm{s}}\right)}, t / \varepsilon\right), \quad \Re\left(t+s+c^{\mathrm{s}}\right) \geq T^{0},
\end{aligned}
$$

with $T^{0}=\ln R_{0} / \omega$ chosen in such a way that $X^{\mathrm{u}}(t, s), X^{\mathrm{s}}(t, s)$ belong to $W$ for the range of parameters $(t, s)$ of $(3.13)$ and (3.14).

It is straightforward to see that parameterizations (3.13) and (3.14) satisfy:

$$
x^{*}(t+2 \pi \varepsilon, s)=x^{*}(t, s+2 \pi \varepsilon) \quad *=\mathrm{s}, \mathrm{u} .
$$

as a consequence of the $2 \pi \varepsilon$-periodicity of the change (2.2).

Now, we will choose the constants $c^{\mathrm{s}}, c^{\mathrm{u}}$ in order to establish estimate (2.9). In fact, by hypothesis $H 1, x^{0}(u)$ is a homoclinic connection for the integrable case $(\mu=0)$. It seems natural to choose the constants in such a way that the parameterizations (3.13) and (3.14) are both equal to this one when $\mu=0$.

By the proof of Theorem 2.1, and more precisely by (3.8) and (3.10), the change (2.2) for $\mu=0$ can be written in vector notation as

$$
x=\Psi^{0}(X)=A^{0} \Phi^{0}(X)=A^{0}\left(X+\mathrm{O}\left(X^{2}\right)\right)=A^{0} X+\mathrm{O}\left(X^{2}\right),
$$

so that using (3.12) for $\mu=0$, the unstable manifold for $(t, s)$ as in (3.13) is given in components by

$$
x^{u, 0}(t, s)=\left(\frac{\mathrm{e}^{\omega^{0}\left(t+s+c^{\mathrm{u}}\right)}}{c}, \frac{\omega^{0} \mathrm{e}^{\omega^{0}\left(t+s+c^{\mathrm{u}}\right)}}{c}\right)+\mathrm{O}\left(\mathrm{e}^{2 \omega^{0}\left(t+s+c^{\mathrm{u}}\right)}\right),
$$

where $c$ is the constant that appears in the matrix $A^{0}$ given in (3.8), whereas using (3.11) for $\mu=0$, the stable manifold for the set $(t, s)$ as in $(3.14)$ is given in components by

$$
x^{s, 0}(t, s)=\left(-\frac{c}{2 \omega^{0}} \mathrm{e}^{-\omega^{0}\left(t+s+c^{\mathrm{s}}\right)}, \frac{c}{2} \mathrm{e}^{-\omega^{0}\left(t+s+c^{\mathrm{s}}\right)}\right)+\mathrm{O}\left(\mathrm{e}^{-2 \omega^{0}\left(t+s+c^{\mathrm{s}}\right)}\right) .
$$

On the other hand, since the origin of the unperturbed system is hyperbolic, the asymptotics of the separatrix $x^{0}(t+s)$ near the origin are given by

$$
\begin{array}{ll}
x^{0}(t+s)=\left(k^{\mathrm{u}} \mathrm{e}^{\omega^{0}(t+s)}, \omega^{0} k^{\mathrm{u}} \mathrm{e}^{\omega^{0}(t+s)}\right)+\mathrm{O}\left(\mathrm{e}^{2 \omega^{0}(t+s)}\right), & t+\Re s \rightarrow-\infty, \\
x^{0}(t+s)=\left(k^{\mathrm{s}} \mathrm{e}^{-\omega^{0}(t+s)},-\omega^{0} k^{\mathrm{s}} \mathrm{e}^{-\omega^{0}(t+s)}\right)+\mathrm{O}\left(\mathrm{e}^{-2 \omega^{0}(t+s)}\right), & t+\Re s \rightarrow \infty,
\end{array}
$$

so we fix the constants $c, c^{\mathrm{s}}, c^{\mathrm{u}}$, to satisfy the conditions:

$$
-\frac{c}{2 \omega^{0}} \mathrm{e}^{-\omega^{0} c^{\mathrm{s}}}=k^{\mathrm{s}}, \quad \frac{1}{c} \mathrm{e}^{\omega^{0} c^{\mathrm{u}}}=k^{\mathrm{u}} .
$$

We have some freedom in order to choose the constants. We will fix $c^{\mathrm{s}}=0$, and the rest are then fixed. Introducing $T / 2 \geq \max \left\{T^{0}+c^{\mathrm{u}}, T^{0}\right\}$, the manifolds defined in (3.13) and (3.14) are also defined for $(t, s)$ in (2.7) and (2.8). 
Coming back to the case $\mu \neq 0$, we can use the form (2.3) of the change (2.2) to write the local unstable manifold for $(t, s)$ satisfying $(2.7)$ as

$$
\begin{aligned}
x^{\mathrm{u}}(t, s) & =\Psi\left(\mathrm{e}^{\omega\left(t+s+c^{\mathrm{u}}\right)}, 0, t / \varepsilon\right) \\
& \left.=\Psi^{0}\left(\mathrm{e}^{\omega\left(t+s+c^{\mathrm{u}}\right.}\right), 0\right)+\mu \varepsilon^{p+1} G\left(\Psi^{0}\left(\mathrm{e}^{\omega\left(t+s+c^{\mathrm{u}}\right)}, 0\right), t / \varepsilon\right)+\mathrm{O}\left(\mu \varepsilon^{p+2}\right),
\end{aligned}
$$

and an analogous expression for the local stable one for $(t, s)$ satisfying (2.8).

Since $\omega=\omega^{0}+\mathrm{O}\left(\mu \varepsilon^{p+2}\right)$, we can assume that $\left|\omega-\omega^{0}\right|<1 / 2$, restricting $\mu_{0}$ if necessary, and hence

$$
\left|\mathrm{e}^{\omega^{0}(t+s)}-\mathrm{e}^{\omega(t+s)}\right|<c^{\prime}\left|\omega-\omega^{0}\right| \mathrm{e}^{(t+\Re s) / 2}
$$

for $t+\Re s<-T / 2$, with $c^{\prime}$ depending only on $T$. In this way we now obtain the estimate (2.9) for the local unstable manifold. The estimate (2.9) for the local stable one is obtained analogously.

The asymptotics $(2.10)$ for $W_{\text {loc }}^{*}\left(\gamma_{p}\right)$ are obtained in the same way, using that $\gamma_{p}(\theta)=$ $\Psi(0, \theta)$, as well as Taylor's Theorem.

\section{Proof of Corollary 2.3}

First of all, solving explicitly system (2.4), it is easy to check that the following change of variables

$$
\begin{aligned}
\Omega: U & \longrightarrow \mathrm{C}^{2} \\
X=\left(X_{1}, X_{2}\right) & \longmapsto(S, E)=\left(-\log X_{2} / F^{\prime}\left(X_{1} X_{2}\right), F\left(X_{1} X_{2}\right)\right)
\end{aligned}
$$

defined on the open set $U=\left\{\left(X_{1}, X_{2}\right) \in W: X_{2}>0\right\}$, provides the flow-box coordinates, which are $\mathrm{O}\left(\mu \varepsilon^{p+2}\right)$-close, by (2.5), to the flow box coordinates of the unperturbed case $\mu=0$ :

Lemma 3.6 1. The change (3.15) transforms system (2.4) into system (2.12).

2. The inverse change $\Xi$ is defined in $\mathcal{V}=\Omega(U)$, and is given by

$$
\begin{aligned}
\Xi: \mathcal{V} & \longrightarrow U \\
(S, E) & \longmapsto\left(X_{1}, X_{2}\right)=\left(\mathrm{e}^{F^{\prime}\left(F^{-1}(E)\right) S} \cdot F^{-1}(E), \mathrm{e}^{-F^{\prime}\left(F^{-1}(E)\right) S}\right)
\end{aligned}
$$

3. Both changes of variables given by (3.15) and (3.16) are canonical.

4. Let $\Omega^{0}, \Xi^{0}$ be the changes (3.15) and (3.16) corresponding to the unperturbed system associated to (2.5). Then

$$
\Omega(X)=\Omega^{0}(X)+\mathrm{O}\left(\mu \varepsilon^{p+2}\right), \quad \Xi(S, E)=\Xi^{0}(S, E)+\mathrm{O}\left(\mu \varepsilon^{p+2}\right),
$$

for $X \in U$ and $(S, E) \in \mathcal{V}$. 
The change (2.11) is constructed simply by composing the change (3.15) with the inverse $\Psi^{-1}$ of the change $\Psi$ given by (2.2), restricting $\mathcal{V}$, if necessary. Namely,

$$
(\mathcal{S}(x, t / \varepsilon), \mathcal{E}(x, t / \varepsilon))=\Omega\left(\Psi^{-1}(x, t / \varepsilon)\right) .
$$

With these changes, equalities (2.13) are a consequence of (3.17) and (2.3).

The inverse change is obtained composing the change $\Psi$ with the change (3.16):

$$
\mathcal{X}(S, E, t / \varepsilon)=\Psi(\Xi(S, E), t / \varepsilon),
$$

and satisfies (2.14), also as a consequence of (3.17) and (2.3).

Finally, from the parameterization (3.14) of the 2-dimensional local stable manifold of $\gamma_{p}$ for $(t, s)$ in $(2.8)$, and taking into account that we have chosen $c^{\mathrm{s}}=0$ along the proof of Corollary 2.2, it is clear that

$$
\left(\mathcal{S}\left(x^{\mathrm{s}}(t, s), t / \varepsilon\right), \mathcal{E}\left(x^{\mathrm{s}}(t, s), t / \varepsilon\right)\right)=\Omega\left(0, \mathrm{e}^{-\omega(t+s)}\right)=(t+s, 0),
$$

i.e., we have obtained formulae (2.15).

\section{Proof of the Extension Theorem}

\subsection{Notation}

Along this proof, $s$ is a complex parameter ranging over the strip $|\Im s| \leq a-\varepsilon, t$ is the real time ranging over $|t+\Re s| \leq T$, and $0<\tau=|t+s-a \mathrm{i}|=\left((t+\Re s)^{2}+(\Im s-a)^{2}\right)^{1 / 2}$, $\tau_{0}=\left|t_{0}+s-a \mathrm{i}\right|, \tau_{1}=\left|t_{1}+s-a \mathrm{i}\right|$. For $v(t) \in \mathbf{C}^{2}$, we introduce

$$
|v(t)|_{\tau}=\left|v_{1}(t)\right|+\left|v_{2}(t)\right| \tau
$$

We will denote by $\varepsilon$ and $\mu$ small independent parameters: $0<\varepsilon<\varepsilon_{0},|\mu|<\mu_{0}$, and $K=K\left(a, T, t_{0}\right)$ will denote a generic positive constant independent of $\varepsilon$ and $\mu$.

\subsection{Set up}

To compare the solution $x(t, s)$ of the full system with the homoclinic solution $x^{0}(t+s)$ of the unperturbed system (1.1) we introduce:

$$
\xi(t)=\xi(t, s):=x(t, s)-x^{0}(t+s) .
$$

The system of differential equations satisfied by $\xi(t)$ with respect to the variable $t$ is written in components as:

$$
\begin{aligned}
& \dot{\xi}_{1}=\xi_{2}+\mu \varepsilon^{p} \partial_{2} h^{1}\left(x^{0}(t+s)+\xi, t / \varepsilon\right), \\
& \dot{\xi}_{2}=f\left(x_{1}^{0}(t+s)+\xi_{1}\right)-f\left(x_{1}^{0}(t+s)\right)-\mu \varepsilon^{p} \partial_{1} h^{1}\left(x^{0}(t+s)+\xi, t / \varepsilon\right),
\end{aligned}
$$


and it is standard to write it as:

$$
\dot{\xi}=A(t+s) \xi+\mu \varepsilon^{p} g\left(x^{0}(t+s), t / \varepsilon\right)+F(\xi, t+s, t / \varepsilon),
$$

where $g=\left(\partial_{2} h^{1},-\partial_{1} h^{1}\right)^{T}, A(u)$ is the matrix

$$
A(u)=\left(\begin{array}{cc}
0 & 1 \\
f^{\prime}\left(x_{1}^{0}(u)\right) & 0
\end{array}\right),
$$

and the function $F(\xi, u, t / \varepsilon)$, which depends also on $\mu \varepsilon^{p}$, is given by:

$$
\begin{aligned}
F(\xi, u, t / \varepsilon)= & \left(\begin{array}{c}
0 \\
f\left(x_{1}^{0}(u)+\xi_{1}\right)-f\left(x_{1}^{0}(u)\right)-f^{\prime}\left(x_{1}^{0}(u)\right) \cdot \xi_{1}
\end{array}\right) \\
& +\mu \varepsilon^{p}\left[g\left(x^{0}(u)+\xi, t / \varepsilon\right)-g\left(x^{0}(u), t / \varepsilon\right)\right] .
\end{aligned}
$$

We first look for an equivalent integral equation for the solutions $\xi(t)$ of system (4.2) with initial condition

$$
\xi\left(t_{0}\right)=\mu \varepsilon^{p+1} G\left(x^{0}\left(t_{0}+s\right), t_{0} / \varepsilon\right)+\mathrm{O}\left(\mu \varepsilon^{p+2}\right),
$$

which is the translation of hypothesis $(2.17)$ on $x\left(t_{0}, s\right)$. For this purpose, we first seek a fundamental matrix of the corresponding homogeneous linear system

$$
\frac{\mathrm{d} \xi}{\mathrm{d} u}=A(u) \xi
$$

A solution of (4.5) is simply $\dot{x}^{0}(u)$. Another independent solution can be obtained in the form: $\xi_{1}(u)=x_{2}^{0}(u) \cdot W(u), \xi_{2}=\xi_{1}^{\prime}:=\mathrm{d} \xi_{1} / \mathrm{d} u$ with

$$
W(u)=\int_{b}^{\mathrm{u}} \frac{\mathrm{d} v}{x_{2}^{0}(v)^{2}},
$$

with $b \in \mathbf{C}$ an arbitrary constant, to be chosen later on to satisfy further properties. Introducing:

$$
\begin{aligned}
& \Psi(u)=x_{2}^{0}(u)=\dot{x}_{1}^{0}(u) \\
& \Phi(u)=x_{2}^{0}(u) W(u)=\Psi(u) W(u)
\end{aligned}
$$

it is easy to see that $\Psi(u) \Phi^{\prime}(u)-\Phi(u) \Psi^{\prime}(u)=\left(x_{2}^{0}\right)^{2}(u) W^{\prime}(u)=1$, and that a fundamental matrix of $(4.5)$ is $M(u)$, where:

$$
M(u)=\left(\begin{array}{cc}
\Psi(u) & \Phi(u) \\
\Psi^{\prime}(u) & \Phi^{\prime}(u)
\end{array}\right),
$$

and hence the fundamental solution $\varphi(u, \sigma)$ of $(4.5)$ satisfying $\varphi(u, u)=\mathrm{Id}$ is given by $\varphi(u, \sigma)=M(u) M(\sigma)^{-1}$, where

$$
M(u)^{-1}=\left(\begin{array}{rr}
\Phi^{\prime}(u) & -\Phi(u) \\
-\Psi^{\prime}(u) & \Psi(u)
\end{array}\right) .
$$


Using this fundamental solution of the linear equation (4.5), we can easily write the solution $\xi(t)$ of system (4.2) with initial condition $\xi\left(t_{0}\right)$ as:

$$
\xi(t)=\xi^{1}(t)+M(t+s) \int_{t_{0}}^{t} M(\sigma+s)^{-1} F(\xi(\sigma), \sigma+s, \sigma / \varepsilon) \mathrm{d} \sigma
$$

with

$$
\xi^{1}(t):=M(t+s)\left[M\left(t_{0}+s\right)^{-1} \xi\left(t_{0}\right)+\mu \varepsilon^{p} \int_{t_{0}}^{t} M(\sigma+s)^{-1} g\left(x^{0}(\sigma+s), \sigma / \varepsilon\right) \mathrm{d} \sigma\right] .
$$

By hypothesis $H 3, g$ has zero mean (with respect to $\theta$ ), and consequently there exists $G(x, \theta)$ with zero mean such that $\partial_{\theta} G=g(G$ is already introduced in formulae $(2.1))$, and therefore $\mathcal{G}(x, \theta)$ such that $\partial_{\theta} \mathcal{G}=G$. Introducing

$$
m(u, \theta):=M(u)^{-1} \mathcal{G}\left(x^{0}(u), \theta\right)
$$

the expression above for $\xi^{1}(t)$ takes the form

$$
\xi^{1}(t)=M(t+s)\left[M\left(t_{0}+s\right)^{-1} \xi\left(t_{0}\right)+\mu \varepsilon^{p} \int_{t_{0}}^{t} \partial_{\theta}^{2} m(\sigma+s, \sigma / \varepsilon) \mathrm{d} \sigma\right] .
$$

Using the identity

$$
\partial_{\theta}^{2} m(\sigma+s, \sigma / \varepsilon)-\varepsilon^{2} m^{\prime \prime}(\sigma+s, \sigma / \varepsilon)=\frac{\mathrm{d}}{\mathrm{d} \sigma}\left[\varepsilon \partial_{\theta} m(\sigma+s, \sigma / \varepsilon)-\varepsilon^{2} m^{\prime}(\sigma+s, \sigma / \varepsilon)\right],
$$

where $m^{\prime}$ denotes $\partial m / \partial u$, as well as $\partial_{\theta} m(u, \theta)=M(u)^{-1} G\left(x^{0}(u), \theta\right)$, we can finally write $\xi^{1}(t)$ as

$$
\begin{aligned}
\xi^{1}(t)= & \mu \varepsilon^{p+1} G\left(x^{0}(t+s), t / \varepsilon\right) \\
& +M(t+s)\left[M\left(t_{0}+s\right)^{-1}\left(\xi\left(t_{0}\right)-\mu \varepsilon^{p+1} G\left(x^{0}\left(t_{0}+s\right), t_{0} / \varepsilon\right)\right)\right. \\
& \left.-\mu \varepsilon^{p+2}\left(m^{\prime}(t+s, t / \varepsilon)-m^{\prime}\left(t_{0}+s, t_{0} / \varepsilon\right)-\int_{t_{0}}^{t} m^{\prime \prime}(\sigma+s, \sigma / \varepsilon) \mathrm{d} \sigma\right)\right] .
\end{aligned}
$$

\subsection{Preliminary bounds}

Now we have a suitable expression (4.8), (4.10) for $\xi$ to carry out an iterative process. Before proceeding with it, we need to bound the fundamental matrix $M(u)$, as well as the functions $f, g$ and $F$.

To get a well behaved fundamental matrix $M(u)$ near the singularity of $x^{0}(u)$, it is very important to choose appropriately the parameter $b$ that is still free in the definition (4.6) of $W(u)$.

We will consider first the case $0 \leq \Im u \leq a$. On the upper boundary of this strip, by hypothesis $H 2, x_{2}^{0}(u)$ has a pole of order $r(r \geq 1)$ at the point $u=a \mathrm{i}$ :

$$
x_{2}^{0}(u)=\dot{x}_{1}^{0}(u)=\frac{\mathrm{C}}{(u-a \mathrm{i})^{r}}(1+\mathrm{O}(u-a \mathrm{i})), \quad \mathrm{C} \neq 0,
$$


and because of this, we will choose precisely $b=a$ i. In this way, $W(u)$ has a zero of multiplicity $2 r+1$ at $u=a \mathrm{i}, y_{0}(u) \cdot W(u)$ has a zero of multiplicity $r+1$ at $u=a \mathrm{i}$, and hence

$$
\begin{aligned}
\Psi(u) & =\frac{\mathrm{C}}{(u-a \mathrm{i})^{r}}(1+\mathrm{O}(u-a \mathrm{i})), \\
\Phi(u) & =\frac{1}{(2 r+1) \mathrm{C}}(u-a \mathrm{i})^{r+1}(1+\mathrm{O}(u-a \mathrm{i})),
\end{aligned}
$$

and as a consequence, the fundamental matrix (4.7) behaves near the pole as:

$$
M(u) \sim\left(\begin{array}{cc}
\frac{\mathrm{C}}{(u-a \mathrm{i})^{r}} & \frac{1}{(2 r+1) \mathrm{C}}(u-a \mathrm{i})^{r+1} \\
\frac{-r \mathrm{C}}{(u-a \mathrm{i})^{r+1}} & \frac{(r+1)}{(2 r+1) \mathrm{C}}(u-a \mathrm{i})^{r}
\end{array}\right)
$$

From the expressions above, next lemma follows directly.

Lemma 4.1 For $|t+\Re s|,\left|t_{1}+\Re s\right| \leq T, 0 \leq \Im s<a$, the following bounds hold:

$$
\begin{aligned}
\left|\Psi^{(k)}(t+s)\right| \leq \frac{K}{\tau^{r+k}}, & \left|\Phi^{(k)}(t+s)\right| \leq K \tau^{r+1-k}, \quad k=0,1,2, \\
|M(t+s) v(t)|_{\tau} & \leq K\left(\frac{\left|v_{1}(t)\right|}{\tau^{r}}+\tau^{r+1}\left|v_{2}(t)\right|\right), \\
\left|M(t+s) M\left(t_{1}+s\right)^{-1} v\left(t_{1}\right)\right|_{\tau} & \leq K\left|v\left(t_{1}\right)\right|_{\tau_{1}}\left(\frac{\tau_{1}^{r}}{\tau^{r}}+\frac{\tau^{r+1}}{\tau_{1}^{r+1}}\right),
\end{aligned}
$$

for every $v(t), v\left(t_{1}\right) \in \mathbf{C}^{2}$, where $|v(t)|_{\tau}:=\left|v_{1}(t)\right|+\left|v_{2}(t)\right| \tau, \tau:=|t+s-a \mathrm{i}|$, and $\left|v\left(t_{1}\right)\right|_{\tau_{1}}:=\left|v_{1}\left(t_{1}\right)\right|+\left|v_{2}\left(t_{1}\right)\right| \tau_{1}, \tau_{1}:=\left|t_{1}+s-a \mathrm{i}\right|$, as introduced in (4.1).

To bound $f, g$ and $F$ on $x=x^{0}(t+s)$, we rely strongly on hypotheses $H 2$ and $H 3$. By the (trigonometric) polynomial character of $f$ (see remark $R 2$ ), and due to the fact that $f\left(x_{1}^{0}(u)\right)=\ddot{x}_{1}^{0}(u)$ has a pole of order $r+1$ at $u=a$ i, we get for $|t+\Re s| \leq T$, $0 \leq \Im s \leq a$ :

$$
\left|f^{(N)}\left(x_{1}^{0}(t+s)\right)\right| \leq \frac{K}{\tau^{r+1-N(r-1)}}=\frac{K}{\tau^{2-(N-1)(r-1)}}, \quad N \geq 0 .
$$

By hypothesis $H 3, h^{1}$ is a (trigonometric) polynomial in $x_{1}$ and a polynomial in $x_{2}$, and all the monomials in $x$ of $h^{1}(x, \theta)$ when evaluated on $x=x^{0}(u)$ have at most a pole of order $\ell$ at $u=a$ i. Consequently, on $|t+\Re s| \leq T, 0 \leq \Im s \leq a$,

$$
\left|\partial_{1}^{N_{1}} \partial_{2}^{N_{2}} h^{1}\left(x^{0}(t+s), t / \varepsilon\right)\right| \leq \frac{K}{\tau^{\ell-N_{1}(r-1)-N_{2} r}}, \quad N_{1}, N_{2} \geq 0 .
$$

From the bounds above on the derivatives of $f$ and $h^{1}$, we get readily the following required bounds for $f, g$ and $F$, simply applying Taylor's Theorem, and using notation (4.1). 
Lemma 4.2 For $\xi^{j}=\xi^{j}(t), j=1,2$, such that $\left|\xi^{j}\right|_{\tau} \leq \eta(\tau) \leq \delta / \tau^{r-1}$, with $\delta<1$, the following bounds hold on $|t+\Re s| \leq T, 0 \leq \Im s \leq a$, with $\varepsilon \leq \tau=|t+s-a \mathrm{i}| \leq T$ :

$$
\begin{aligned}
\left|f\left(x_{1}^{0}(t+s)+\xi_{1}^{1}\right)-f\left(x_{1}^{0}(t+s)+\xi_{1}^{2}\right)\right| & \leq \frac{K\left|\xi_{1}^{1}-\xi_{1}^{2}\right|}{\tau^{2}} \\
\left|g\left(x^{0}(t+s)+\xi^{1}, t / \varepsilon\right)-g\left(x^{0}(t+s)+\xi^{2}, t / \varepsilon\right)\right|_{\tau} & \leq \frac{K\left|\xi^{1}-\xi^{2}\right|_{\tau}}{\tau^{\ell-2 r+1}} \\
\left|F\left(\xi^{1}, t+s, t / \varepsilon\right)-F\left(\xi^{2}, t+s, t / \varepsilon\right)\right|_{\tau} & \leq K\left(\frac{\mu \varepsilon^{p}}{\tau^{\ell-2 r+1}}+\frac{\eta(\tau)}{\tau^{2-r}}\right)\left|\xi^{1}-\xi^{2}\right|_{\tau}
\end{aligned}
$$

Finally, we state now the last technical lemma that will be needed later on.

Lemma 4.3 For $t, t_{0}, \beta$ real, s complex, such that

$$
0 \leq \Im s<a, \quad-T \leq t_{0}+\Re s \leq t+\Re s \leq T
$$

let us denote

$$
\rho_{\left[t_{0}, t\right]}^{-\beta}(s):= \begin{cases}\sup \frac{1}{|\sigma+s-a \mathrm{i}|^{\beta}}, & \text { if } \beta \neq 0, \\ \sup |\ln (|\sigma+s-a \mathrm{i}|)|, & \text { if } \beta=0,\end{cases}
$$

where the supremum is taken on $\sigma \in\left[t_{0}, t\right]$.

Then, there exists $K=K\left(a, t_{0}, T, \beta\right)>0$ such that the following inequalities hold:

$$
\begin{aligned}
\int_{t_{0}}^{t} \frac{\mathrm{d} \sigma}{|\sigma+s-a \mathrm{i}|^{\beta}} & \leq K \cdot \rho_{\left[t_{0}, t\right]}^{-(\beta-1)}(s) \\
\left|M(t+s) \int_{t_{0}}^{t} M(\sigma+s)^{-1} v(\sigma) \mathrm{d} \sigma\right|_{\tau} & \leq K \mathrm{C}\left(\frac{\rho_{\left[t_{0}, t\right]}^{-(\beta-r-1)}(s)}{\tau^{r}}+\tau^{r+1} \rho_{\left[t_{0}, t\right]}^{-(\beta+r)}(s)\right),
\end{aligned}
$$

where $\tau=|t+s-a \mathrm{i}|$ and $v(t) \in \mathbf{C}^{2}$ is such that

$$
|v(t)|_{\tau}:=\left|v_{1}(t)\right|+\left|v_{2}(t)\right| \tau \leq \mathrm{C} / \tau^{\beta}
$$

The proof of (4.16) is straightforward (and can be found in [DS92, Lemma 7.1] for $\beta=3$ and $a=\pi / 2$ ). Bound (4.17) for functions $v(t) \in \mathbf{C}^{2}$ satisfying (4.18) follows readily from $(4.12)$ and $(4.16)$.

\subsection{Partition of the domain}

The proof of the Extension Theorem, for the moment for $0 \leq \Im s \leq a-\varepsilon$, is based on the following two propositions. In the first one, the solutions of system (4.2) with initial 


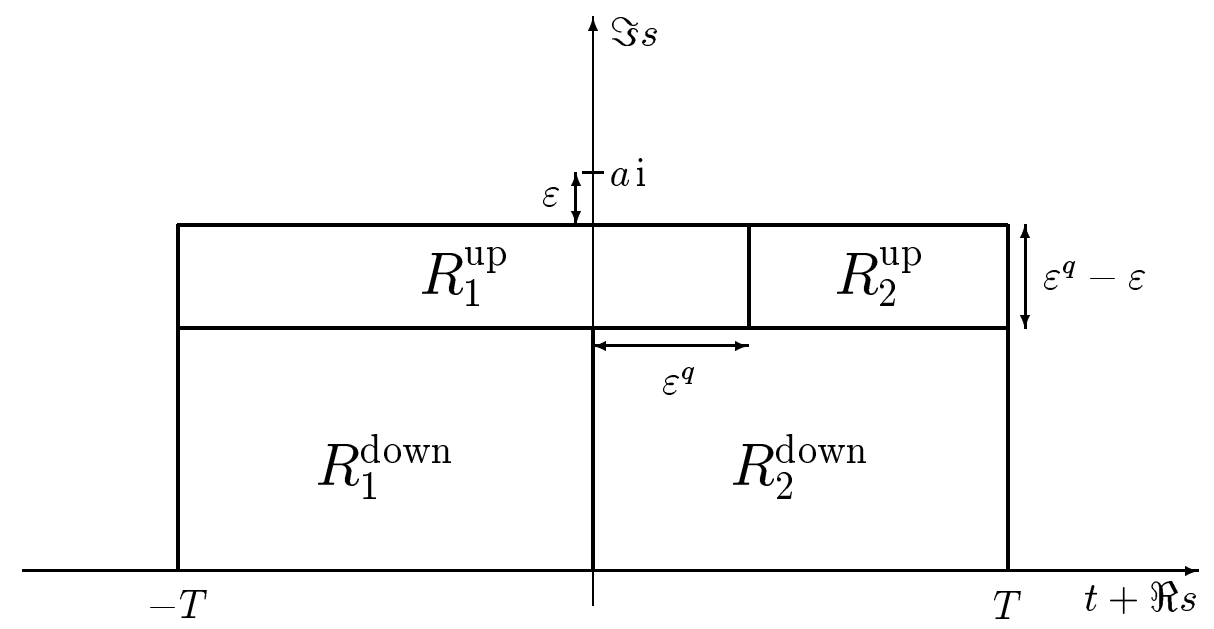

Figure 1: Domains $D_{1}=R_{1}^{\mathrm{up}} \cup R_{1}^{\text {down }}, D_{2}=R_{2}^{\text {up }} \cup R_{2}^{\text {down }}$.

conditions (4.4) will be extended for $(t, s) \in D_{1}:=R_{1}^{\text {up }} \cup R_{1}^{\text {down }}$, where $R_{1}^{\text {up }}, R_{1}^{\text {down }}$ are the rectangles given by the equations (see figure 1 )

$$
\begin{aligned}
R_{1}^{\text {up }}: & -T \leq t+\Re s \leq \varepsilon^{q}, \quad a-\varepsilon^{q} \leq \Im s \leq a-\varepsilon, \\
R_{1}^{\text {down }}: & -T \leq t+\Re s \leq 0, \quad 0 \leq \Im s \leq a-\varepsilon^{q},
\end{aligned}
$$

where $-T:=t_{0}+\Re s$ and $q=(r+1) /(2 r+1)$. Since $r \geq 1$, then $1 / 2<q \leq 2 / 3$. In the second proposition, we take the separation point $t=t_{1}(s)$ :

$$
t_{1}+\Re s:=\left\{\begin{array}{llr}
\varepsilon^{q}, & \text { for } & a-\varepsilon^{q} \leq \Im s \leq a-\varepsilon \\
0, & \text { for } & 0 \leq \Im s \leq a-\varepsilon^{q}
\end{array}\right.
$$

as the initial time, and these solutions of system (4.2) will be extended for $(t, s) \in D_{2}:=$ $R_{2}^{\text {up }} \cup R_{2}^{\text {down }}$, where $R_{2}^{\text {up }}, R_{2}^{\text {down }}$ are the rectangles given by the equations

$$
\begin{aligned}
R_{2}^{\text {up }}: & \varepsilon^{q} \leq t+\Re s \leq T, \quad a-\varepsilon^{q} \leq \Im s \leq a-\varepsilon, \\
R_{2}^{\text {down }}: & 0 \leq t+\Re s \leq T, \quad 0 \leq \Im s \leq a-\varepsilon^{q} .
\end{aligned}
$$

\subsection{First domain}

Proposition 4.4 Given $s \in \mathbf{C}$ such that $0 \leq \Im s \leq a-\varepsilon$, let $\xi(t)=\xi(t, s)$ be a solution of system (4.2) with initial condition (4.4) on $t_{0}=-T-\Re s$. Then, if $\gamma:=p-\ell \geq 0$, the solution $\xi(t)$ can be extended for $t \in\left[t_{0}, t_{1}(s)\right]$, with $t_{1}(s)$ given in (4.19), satisfying there the following estimates:

$$
\begin{aligned}
\left|\xi(t)-\mu \varepsilon^{p+1} G\left(x^{0}(t+s), t / \varepsilon\right)\right|_{\tau} & \leq K \frac{\mu \varepsilon^{\gamma+r+1}}{\tau^{r}} \\
|\xi(t)|_{\tau} & \leq K \mu \varepsilon^{\gamma+1}
\end{aligned}
$$


Proof. We shall use the method of successive approximations. We begin the iteration process with $\xi^{0}(t)=0$, and consider for $n \geq 0$ and $t \in\left[t_{0}, t_{1}(s)\right]$ the recurrence suggested by equation (4.8):

$$
\xi^{n+1}(t)=\xi^{1}(t)+M(t+s) \int_{t_{0}}^{t} M(\sigma+s)^{-1} F\left(\xi^{n}(\sigma), \sigma+s, \sigma / \varepsilon\right) \mathrm{d} \sigma .
$$

The first iterate is $\xi^{1}(t)$, as given by (4.10). The main idea is to check that $\xi^{1}$ satisfies the required bound (4.20), and that the successive iterates are small enough to preserve it for the limit $\xi$.

There are several terms in the expression (4.10) of $\xi^{1}(t)$. From the initial condition (4.4) and the fact that $t_{0}+s=-T+\Im s$ is far from the singularity $a$ i, we get

$$
M\left(t_{0}+s\right)^{-1}\left(\xi\left(t_{0}\right)-\mu \varepsilon^{p+1} G\left(x^{0}\left(t_{0}+s\right), t_{0} / \varepsilon\right)\right)=\mathrm{O}\left(\mu \varepsilon^{p+2}\right),
$$

and consequently, using (4.11), we can bound one term of (4.10):

$$
\left|M(t+s) M\left(t_{0}+s\right)^{-1}\left(\xi\left(t_{0}\right)-\mu \varepsilon^{p+1} G\left(x^{0}\left(t_{0}+s\right), t_{0} / \varepsilon\right)\right)\right|_{\tau} \leq K \mu \varepsilon^{p+2}\left[\frac{1}{\tau^{r}}+\tau^{r+1}\right] .
$$

For the terms involving $m$, we use definition (4.9): $m=M^{-1} \mathcal{G}$, where for simplicity $\mathcal{G}$ denotes $\mathcal{G}\left(x^{0}(u), \theta\right)$, as well as the fact that $\left(M^{-1}\right)^{\prime}=-M^{-1} A$, where ' denotes $\partial / \partial u$, to get:

$$
m^{\prime}=M^{-1}\left(\mathcal{G}^{\prime}-A \mathcal{G}\right)=: M^{-1} \mathcal{G}_{1}, \quad m^{\prime \prime}=M^{-1}\left(\mathcal{G}_{1}^{\prime}-A \mathcal{G}_{1}\right)=: M^{-1} \mathcal{G}_{2} .
$$

Now, one has to take into account that $G$ and $\mathcal{G}$ have the same kind of bounds (4.14) as $g=\left(\partial_{2} h^{1},-\partial_{1} h^{1}\right)^{T}$, as well as the definitions of $\mathcal{G}_{1}$ and $\mathcal{G}_{2}$ and the form (4.3) of the matrix $A$ to get the bounds:

$$
|G|_{\tau} \leq \frac{K}{\tau^{\ell-r}}, \quad|\mathcal{G}|_{\tau} \leq \frac{K}{\tau^{\ell-r}}, \quad\left|\mathcal{G}_{1}\right|_{\tau} \leq \frac{K}{\tau^{\ell-r+1}}, \quad\left|\mathcal{G}_{2}\right|_{\tau} \leq \frac{K}{\tau^{\ell-r+2}},
$$

for $|t+\Re s| \leq T, 0 \leq \Im s<a$. Here, $|G|_{\tau}$ denotes $\left|G_{1}\left(x^{0}(t+s), \theta\right)\right|+\left|G_{2}\left(x^{0}(t+s), \theta\right)\right| \tau$, according to notation (4.1). Analogously for $|\mathcal{G}|_{\tau},\left|\mathcal{G}_{1}\right|_{\tau}$, and $\left|\mathcal{G}_{2}\right|_{\tau}$.

Using expressions (4.22) for $m^{\prime}, m^{\prime \prime}$, we now proceed to estimate $\xi^{1}(t)$ given in (4.10). We simply use bounds (4.23), as well as bounds (4.12) and (4.17), with $\beta=\ell-r+2$ and $\mathrm{C}=\mu \varepsilon^{p+2}$, to get

$$
\begin{aligned}
& \left|\xi^{1}(t)-\mu \varepsilon^{p+1} G\left(x^{0}(t+s), t / \varepsilon\right)\right|_{\tau} \\
& \quad \leq K \mu \varepsilon^{p+2}\left[\frac{1}{\tau^{r}}+\tau^{r+1}+\frac{1}{\tau^{\ell-r+1}}+\frac{1}{\tau^{r}}+\tau^{r+1}+\frac{\rho_{0}^{-(\ell-2 r+1)}}{\tau^{r}}+\tau^{r+1} \rho_{0}^{-(\ell+2)}\right],
\end{aligned}
$$

where $\rho_{0}^{-(\ell-2 r+1)}, \rho_{0}^{-(\ell+2)}$ denote, respectively, $\rho_{\left[t_{0}, t\right]}^{-(\ell-2 r+1)}(s), \rho_{\left[t_{0}, t\right]}^{-(\ell+2)}(s)$. Depending on the sign of $\ell-2 r+1$ and $t+\Re s$, the term $\rho_{0}^{-(\ell-2 r+1)}$ has different estimates. Thus, for 
$(t, s) \in D_{1}$ and $t+\Re s<0$

$$
\rho_{0}^{-(\ell+2)} \leq \tau^{-(\ell+2)}, \quad \rho_{0}^{-(\ell-2 r+1)} \leq \begin{cases}\tau^{-(\ell-2 r+1)}, & \ell-2 r+1>0 \\ |\ln \tau|, & \ell-2 r+1=0 \\ K, & \ell-2 r+1 \leq 0\end{cases}
$$

whereas for $(t, s) \in D_{1}$ and $t+\Re s \geq 0$

$$
\rho_{0}^{-(\ell+2)} \leq \varepsilon^{-(\ell+2)}, \quad \rho_{0}^{-(\ell-2 r+1)} \leq\left\{\begin{array}{ll}
\varepsilon^{-(\ell-2 r+1)}, & \ell-2 r+1>0 \\
|\ln \varepsilon|, & \ell-2 r+1=0 \\
K, & \ell-2 r+1 \leq 0
\end{array},\right.
$$

and $\tau^{2 r+1} \leq \varepsilon^{r+1}$. Hence, in all the domain $D_{1}$ we have

$$
\tau^{2 r+1} \rho_{0}^{-(\ell+2)} \leq \frac{1}{\varepsilon^{\ell-r+1}}, \quad \rho_{0}^{-(\ell-2 r+1)} \leq K\left(\frac{|\ln \varepsilon|}{\varepsilon^{\ell-2 r+1}}\right) .
$$

Using these bounds above, jointly with the fact that $\ell \geq r-1$ (see remark $R 3$ ), we obtain the following estimate for $\xi^{1}(t)$ :

$$
\begin{aligned}
\mid \xi^{1}(t)-\mu \varepsilon^{p+1} G( & \left.x^{0}(t+s), t / \varepsilon\right)\left.\right|_{\tau} \tau^{r} \\
& \leq K \mu \varepsilon^{p+2}\left[1+\tau^{2 r+1}+\frac{1}{\tau^{\ell-2 r+1}}+\rho_{0}^{-(\ell-2 r+1)}+\tau^{2 r+1} \rho_{0}^{-(l+2)}\right] \\
& \leq K \mu \varepsilon^{p+2}\left[1+\frac{|\ln \varepsilon|}{\varepsilon^{\ell-2 r+1}}+\frac{1}{\varepsilon^{\ell-r+1}}\right] \leq K \mu \varepsilon^{\gamma+r+1} .
\end{aligned}
$$

Introducing the norm $\|\xi\|_{r}:=\sup |\xi(t)|_{\tau} \tau^{r}$, where the supremum is taken on $t \in$ $\left[t_{0}, t_{1}(s)\right]$, the estimate above for $\xi^{1}$ can be expressed as

$$
\left\|\xi^{1}-\mu \varepsilon^{p+1} G\right\|_{r} \leq K \mu \varepsilon^{\gamma+r+1} .
$$

We now proceed by induction. Assuming that for $k=1, \ldots, n$,

$$
\left\|\xi^{k}-\mu \varepsilon^{p+1} G\right\|_{r} \leq K \mu \varepsilon^{\gamma+r+1},
$$

estimate (4.23) on $G$ gives

$$
\left|\xi^{k}(t)\right|_{\tau} \leq \eta(\tau):=K \frac{\mu \varepsilon^{p+1}}{\tau^{\ell-r}}+K \frac{\mu \varepsilon^{\gamma+r+1}}{\tau^{r}}
$$

Since $\gamma=p-\ell \geq 0$ and $\varepsilon \leq \tau$ on $D_{1}$, we can bound $\eta(\tau)$ as $\eta(\tau) \leq K \mu \varepsilon^{\gamma} / \tau^{r-1}$, and hence we can apply estimate (4.15) to $F\left(\xi^{n}(t), t+s, t / \varepsilon\right)-F\left(\xi^{n-1}(t), t+s, t / \varepsilon\right)$ :

$$
\begin{aligned}
\left|F\left(\xi^{n}(t), t+s, t / \varepsilon\right)-F\left(\xi^{n-1}(t), t+s, t / \varepsilon\right)\right|_{\tau} & \\
& \leq K \mu\left(\frac{\varepsilon^{p}}{\tau^{\ell-2 r+1}}+\frac{\varepsilon^{p+1}}{\tau^{\ell-2 r+2}}+\frac{\varepsilon^{\gamma+r+1}}{\tau^{2}}\right)\left|\xi^{n}-\xi^{n-1}\right|_{\tau} \\
& \leq K \mu\left(\frac{\varepsilon^{p}}{\tau^{\ell-r+1}}+\frac{\varepsilon^{p+1}}{\tau^{\ell-r+2}}+\frac{\varepsilon^{\gamma+r+1}}{\tau^{r+2}}\right)\left\|\xi^{n}-\xi^{n-1}\right\|_{r}
\end{aligned}
$$


Applying thrice Lemma 4.3 with $\beta=\ell-r+1$ and $\mathrm{C}=\varepsilon^{p}, \beta=\ell-r+2$ and $\mathrm{C}=\varepsilon^{p+1}$, $\beta=r+2$ and $\mathrm{C}=\varepsilon^{\gamma+r+1}$, respectively, we obtain

$$
\begin{aligned}
\left|M(t+s) \int_{t_{0}}^{t} M(\sigma+s)^{-1}\left(F\left(\xi^{n}(\sigma), \sigma+s, \sigma / \varepsilon\right)-F\left(\xi^{n-1}(\sigma), \sigma+s, \sigma / \varepsilon\right)\right) \mathrm{d} \sigma\right|_{\tau} \\
\leq \quad\left[K \mu \varepsilon^{p}\left(\frac{\rho_{0}^{-(\ell-2 r)}}{\tau^{r}}+\tau^{r+1} \rho_{0}^{-(\ell+1)}\right)+K \mu \varepsilon^{p+1}\left(\frac{\rho_{0}^{-(\ell-2 r+1)}}{\tau^{r}}+\tau^{r+1} \rho_{0}^{-(\ell+2)}\right)\right. \\
\left.\quad+K \mu \varepsilon^{\gamma+r+1}\left(\frac{\rho_{0}^{-(1)}}{\tau^{r}}+\tau^{r+1} \rho_{0}^{-(2 r+2)}\right)\right]\left\|\xi^{n}-\xi^{n-1}\right\|_{r} .
\end{aligned}
$$

Finally, multiplying by $\tau^{r}$ and taking supremum, and using inequalities like those of (4.24), we arrive at

$$
\begin{aligned}
\left\|\xi^{n+1}-\xi^{n}\right\|_{r} \leq & K \mu\left[\varepsilon^{p}\left(1+\frac{|\ln \varepsilon|}{\varepsilon^{\ell-2 r}}+\frac{1}{\varepsilon^{\ell-r}}\right)+\varepsilon^{p+1}\left(1+\frac{|\ln \varepsilon|}{\varepsilon^{\ell-2 r+1}}+\frac{1}{\varepsilon^{\ell-r+1}}\right)\right. \\
& \left.+\varepsilon^{\gamma+r+1}\left(\frac{1}{\varepsilon}+\frac{1}{\varepsilon^{r+1}}\right)\right]\left\|\xi^{n}-\xi^{n-1}\right\|_{r} \\
\leq & K \mu\left(\varepsilon^{p}+\varepsilon^{\gamma}\right)\left\|\xi^{n}-\xi^{n-1}\right\|_{r} .
\end{aligned}
$$

If we choose now $\mu_{0}$ small enough, and $\gamma \geq 0$, (and hence $p \geq 0$ ) it follows that for $n \geq 1,|\mu| \leq \mu_{0}$

$$
\begin{aligned}
\left\|\xi^{n}-\mu \varepsilon^{p+1} G\right\|_{r} & \leq 2\left\|\xi^{1}-\mu \varepsilon^{p+1} G\right\|_{r} \leq K \mu \varepsilon^{\gamma+r+1} \\
\left\|\xi^{n+1}-\xi^{n}\right\|_{r} & \leq \frac{1}{2}\left\|\xi^{n}-\xi^{n-1}\right\|_{r}
\end{aligned}
$$

and consequently $\left(\xi^{n}\right)_{n \geq 0}$ converges uniformly on $t \in\left[t_{0}, t_{1}(s)\right]$ to the solution $\xi(t)$ of system (4.2), satisfying there

$$
\left\|\xi-\mu \varepsilon^{p+1} G\right\|_{r} \leq K \mu \varepsilon^{\gamma+r+1}
$$

and hence estimate (4.20). Estimate (4.21) is then an easy consequence of estimate (4.23) on $G$ and the fact that $\varepsilon \leq \tau$ and $\ell \geq 0$ :

$$
|\xi(t)|_{\tau} \leq K \frac{\mu \varepsilon^{p+1}}{\tau^{\ell-r}}+K \frac{\mu \varepsilon^{\gamma+r+1}}{\tau^{r}} \leq K \frac{\mu \varepsilon^{p+1} \tau^{r}}{\varepsilon^{\ell}}+K \mu \varepsilon^{\gamma+1} \leq K \mu \varepsilon^{\gamma+1} .
$$

\subsection{Second domain}

On the final point $t_{1}(s)$, estimate $(4.20)$ provided by Proposition 4.4 reads as:

$$
\left|\xi\left(t_{1}\right)-\mu \varepsilon^{p+1} G\left(x^{0}\left(t_{1}+s\right), t_{1} / \varepsilon\right)\right|_{\tau_{1}} \leq K \frac{\mu \varepsilon^{\gamma+1+r}}{\tau_{1}^{r}}
$$

were $\tau_{1}=\left|t_{1}+s-a \mathrm{i}\right|$. 
Proposition 4.5 Given $s \in \mathbf{C}$ such that $0 \leq \Im s \leq a-\varepsilon$, let $\xi(t)=\xi(t, s)$ be a solution of system (4.2) with initial condition (4.25) on $t_{1}=t_{1}(s)$ as given in (4.19). Then, if $\gamma:=p-\ell \geq 0$, the solution $\xi(t)$ can be extended for $t \in\left[t_{1}(s), T-\Re s\right]$, satisfying there the following estimate:

$$
|\xi(t)|_{\tau} \leq K \mu \varepsilon^{\gamma} \tau^{r+1}
$$

Proof. We shall use exactly the same method of successive approximations as in Proposition 4.4 , but replacing the initial condition $t_{0}$ by $t_{1}$ :

$$
\xi^{n+1}(t)=\xi^{1}(t)+M(t+s) \int_{t_{1}}^{t} M^{-1}(\sigma+s) F\left(\xi^{n}(\sigma), \sigma+s, \sigma / \varepsilon\right) \mathrm{d} \sigma,
$$

for $t \in\left[t_{1}(s), T-\Re s\right]$. The first iterate is $\xi^{1}(t)$ as given by expression (4.10), but with $t_{1}$ instead of $t_{0}$.

From initial condition (4.25) and estimate (4.12), we can bound one term of the expression $(4.10)$ of $\xi^{1}(t)$ :

$$
\left|M(t+s) M\left(t_{1}+s\right)^{-1}\left(\xi^{1}\left(t_{1}\right)-\mu \varepsilon^{p+1} G\left(x^{0}\left(t_{1}+s\right), t_{1} / \varepsilon\right)\right)\right|_{\tau} \leq K \mu \varepsilon^{\gamma+r+1}\left(\frac{1}{\tau^{r}}+\frac{\tau^{r+1}}{\tau_{1}^{2 r+1}}\right) .
$$

We use the expressions (4.22) of $\mathrm{m}^{\prime}, \mathrm{m}^{\prime \prime}$, for the terms involving them. With the help of bounds (4.23), we apply (4.12) for the terms involving $m^{\prime}$ in expression (4.10) of $\xi^{1}(t)$, and estimate (4.17) with $\beta=\ell-r+2$ and $\mathrm{C}=\mu \varepsilon^{p+2}$ for the term involving $m^{\prime \prime}$ to get

$$
\begin{aligned}
\left|\xi^{1}-\mu \varepsilon^{p+1} G\right|_{\tau} \leq & K \mu \varepsilon^{\gamma+r+1}\left(\frac{1}{\tau^{r}}+\frac{\tau^{r+1}}{\tau_{1}^{2 r+1}}\right)+K \frac{\mu \varepsilon^{p+2}}{\tau^{\ell-r+1}} \\
& +K \frac{\mu \varepsilon^{p+2}}{\tau_{1}^{\ell-r+1}}\left(\frac{\tau_{1}^{r}}{\tau^{r}}+\frac{\tau^{r+1}}{\tau_{1}^{r+1}}\right)+K \mu \varepsilon^{p+2}\left(\frac{\rho_{1}^{-(\ell-2 r+1)}}{\tau^{r}}+\tau^{r+1} \rho_{1}^{-(\ell+2)}\right),
\end{aligned}
$$

where $\rho_{1}^{-(\ell-2 r+1)}, \rho_{1}^{-(\ell+2)}$ denote, respectively, $\rho_{\left[t_{1}, t\right]}^{-(\ell-2 r+1)}(s), \rho_{\left[t_{1}, t\right]}^{-(\ell+2)}(s)$. Now, for $(t, s) \in$ $D_{2}$, these terms can be bounded as

$$
\rho_{1}^{\beta} \leq \begin{cases}\tau^{\beta}, & \beta>0 \\ \left|\ln \tau_{1}\right|, & \beta=0 \\ \tau_{1}^{\beta}, & \beta<0\end{cases}
$$

and consequently, we obtain the following estimate for $\xi^{1}(t)$ :

$$
\begin{aligned}
\frac{\left|\xi^{1}-\mu \varepsilon^{p+1} G\right|_{\tau}}{\tau^{r+1}} \leq & K \mu \varepsilon^{\gamma+r+1}\left(\frac{1}{\tau^{2 r+1}}+\frac{1}{\tau_{1}^{2 r+1}}\right)+K \frac{\mu \varepsilon^{p+2}}{\tau^{\ell+2}} \\
& +K \frac{\mu \varepsilon^{p+2}}{\tau_{1}^{\ell-r+1}}\left(\frac{\tau_{1}^{r}}{\tau^{2 r+1}}+\frac{1}{\tau_{1}^{r+1}}\right)+K \mu \varepsilon^{p+2}\left(\frac{\rho_{1}^{-(\ell-2 r+1)}}{\tau^{2 r+1}}+\rho_{1}^{-(\ell+2)}\right) \\
\leq & K \mu \varepsilon^{\gamma}
\end{aligned}
$$


where we have used that $\varepsilon<\varepsilon^{q} \leq \tau_{1} \leq \tau$, and $q=(r+1) /(2 r+1)$. Bound (4.23) on $G$, jointly with $\tau \geq \varepsilon$, gives at last

$$
\left|\xi^{1}(t)\right|_{\tau} \tau^{-(r+1)} \leq K \mu \varepsilon^{\gamma}+K \frac{\mu \varepsilon^{p+1}}{\tau^{\ell+1}} \leq K \mu \varepsilon^{\gamma} .
$$

In view of this bound, we define the norm $\|\xi\|_{-(r+1)}:=\sup |\xi(t)|_{\tau} \tau^{-(r+1)}$, where the supremum is taken on $t \in\left[t_{1}(s), T-\Re s\right]$. With this new terminology we have proved that

$$
\left\|\xi^{1}\right\|_{-(r+1)} \leq K \mu \varepsilon^{\gamma} .
$$

We now proceed by induction. Assuming that for $k=1, \ldots, n$,

$$
\left\|\xi^{k}\right\|_{-(r+1)} \leq K \mu \varepsilon^{\gamma},
$$

and consequently

$$
\left|\xi^{k}(t)\right|_{\tau} \leq \eta(\tau):=K \mu \varepsilon^{\gamma} \tau^{r+1},
$$

then obviously $\eta(\tau) \leq K \mu \varepsilon^{\gamma} / \tau^{r-1}$, and we apply estimate (4.15) to get

$$
\begin{aligned}
& \left|F\left(\xi^{n}(t), t+s, t / \varepsilon\right)-F\left(\xi^{n-1}(t), t+s, t / \varepsilon\right)\right|_{\tau} \\
& \quad \leq K\left(\frac{\mu \varepsilon^{p}}{\tau^{\ell-2 r+1}}+\frac{\mu \varepsilon^{\gamma} \tau^{r+1}}{\tau^{2-r}}\right)\left|\xi^{n}-\xi^{n-1}\right|_{\tau} \\
& \quad \leq K\left(\frac{\mu \varepsilon^{p}}{\tau^{\ell-3 r}}+\mu \varepsilon^{\gamma} \tau^{3 r}\right)\left\|\xi^{n}-\xi^{n-1}\right\|_{-(r+1)} \leq K \mu \varepsilon^{\gamma} \tau^{3 r}\left\|\xi^{n}-\xi^{n-1}\right\|_{-(r+1)}
\end{aligned}
$$

Applying estimate (4.17) of Lemma 4.3 with $\beta=-3 r$ and $\mathrm{C}=\mu \varepsilon^{\gamma}$, we obtain

$$
\begin{aligned}
& \mid M(t+s) \int_{t_{1}}^{t} M(\sigma+s)^{-1}(\left.F\left(\xi^{n}(\sigma), \sigma+s, \sigma / \varepsilon\right)-F\left(\xi^{n-1}(\sigma), \sigma+s, \sigma / \varepsilon\right)\right)\left.\mathrm{d} \sigma\right|_{\tau} \\
& \leq K \mu \varepsilon^{\gamma}\left(\frac{\rho_{1}^{4 r+1}}{\tau^{r}}+\tau^{r+1} \rho_{1}^{2 r}\right)\left\|\xi^{n}-\xi^{n-1}\right\|_{-(r+1)},
\end{aligned}
$$

and using inequalities (4.27), we arrive at

$$
\begin{aligned}
\left|\xi^{n+1}(t)-\xi^{n}(t)\right|_{\tau} \tau^{-(r+1)} & \leq K \mu \varepsilon^{\gamma}\left(\frac{\rho_{1}^{4 r+1}}{\tau^{2 r+1}}+\rho_{1}^{2 r}\right)\left\|\xi^{n}-\xi^{n-1}\right\|_{-(r+1)} \\
& \leq K \mu \varepsilon^{\gamma}\left\|\xi^{n}-\xi^{n-1}\right\|_{-(r+1)} .
\end{aligned}
$$

Since $\gamma \geq 0$, choosing now $\mu_{0}$ small enough, it follows by induction that for $n \geq 1$, $|\mu| \leq \mu_{0}$,

$$
\begin{aligned}
\left\|\xi^{n}\right\|_{-(r+1)} & \leq K \mu \varepsilon^{\gamma} \\
\left\|\xi^{n+1}-\xi^{n}\right\|_{-(r+1)} & \leq \frac{1}{2}\left\|\xi^{n}-\xi^{n-1}\right\|_{-(r+1)},
\end{aligned}
$$

and thus $\left(\xi^{n}\right)_{n \geq 0}$ converges uniformly on $t \in\left[t_{1}(s), T-\Re s\right]$ to the solution $\xi(t)$ of system (4.2), satisfying there the required estimate (4.26). 


\subsection{Proof of Theorem 2.4}

First consider $0 \leq \Im s \leq a-\varepsilon$. Putting Propositions 4.4 and 4.5 together, from $|\xi(t)|_{\tau}=$ $\left|\xi_{1}(t)\right|+\left|\xi_{2}(t)\right| \tau$, we immediately obtain $\xi(t)=\mathrm{O}\left(\mu \varepsilon^{\gamma}\right)$, and the Extension Theorem 2.4 is proved.

For $-a+\varepsilon \leq \Im s \leq 0$ we only have to choose $b=-a$ i in the definition of $W(u)$, in order to get a second solution $\Phi(u)$ of the linear system (4.5) having a zero of multiplicity $r+1$ at $u=-a$ i. Propositions 4.4 and 4.5 follow exactly in the same way for $-a+\varepsilon \leq$ $\Im s \leq 0$, as well as the Extension Theorem.

Remark 4.6 The proof given here can be generalized for perturbations $h^{1}(x, \theta)$ not necessarily polynomials, but satisfying (4.14). In this case, $\gamma$ has to be chosen as $\gamma=$ $\min (p-r+1, p-\ell)$, and the condition $\gamma \geq 0$ is also required.

\section{Proof of Propositions 2.6 and 2.7}

\section{Proof of Proposition 2.6}

1. Using (2.15) and (2.13) in the definition (2.20) of $\mathcal{E}^{\mathrm{u}}(s)$, it follows that

$$
\mathcal{E}^{\mathrm{u}}(s)=\mathcal{E}\left(x^{\mathrm{u}}(t, s), t / \varepsilon\right)-\mathcal{E}\left(x^{\mathrm{s}}(t, s), t / \varepsilon\right)=h^{0}\left(x^{\mathrm{u}}(t, s)\right)-h^{0}\left(x^{\mathrm{s}}(t, s)\right)+\mathrm{O}\left(\mu \varepsilon^{p+1}\right),
$$

for $t$ such that $T / 2 \leq t+\Re s \leq T$, in such a way that both manifolds $x^{\mathrm{u}}(t, s)$ and $x^{\mathrm{s}}(t, s)$ belong to $\mathcal{U}$. Since $\mathcal{E}^{\mathrm{u}}(s)$ does not depend on $t$, from now on we take for example $t=T_{s} / 2$, with $T_{s}:=T-\Re s$.

For $*=\mathrm{s}, \mathrm{u}$, let us introduce the functions

$$
\Delta^{*}(t, s)=h^{0}\left(x^{*}(t, s)\right)-h^{0}\left(\gamma_{p}(t / \varepsilon)\right) .
$$

It is straightforward to check that:

$$
\begin{aligned}
& \lim _{t \rightarrow+\infty} \Delta^{\mathrm{s}}(t, s)=0, \lim _{t \rightarrow-\infty} \Delta^{\mathrm{u}}(t, s)=0, \\
& \partial_{t} \Delta^{*}(t, s)= \mu \varepsilon^{p}\left(\left\{h^{0}, h^{1}\right\}\left(x^{*}(t, s), t / \varepsilon\right)-\left\{h^{0}, h^{1}\right\}\left(\gamma_{p}(t / \varepsilon), t / \varepsilon\right)\right), \\
& h^{0}\left(x^{\mathrm{u}}(t, s)\right)-h^{0}\left(x^{\mathrm{s}}(t, s)\right)=\Delta^{\mathrm{u}}(t, s)-\Delta^{\mathrm{s}}(t, s),
\end{aligned}
$$

and thus

$$
\begin{array}{r}
h^{0}\left(x^{\mathrm{u}}\left(T_{s} / 2, s\right)\right)-h^{0}\left(x^{\mathrm{s}}\left(T_{s} / 2, s\right)\right)=\mu \varepsilon^{p}\left[\int_{-\infty}^{T_{s} / 2}\left(\left\{h^{0}, h^{1}\right\}\left(x^{\mathrm{u}}, t / \varepsilon\right)-\left\{h^{0}, h^{1}\right\}\left(\gamma_{p}, t / \varepsilon\right)\right) \mathrm{d} t\right. \\
\left.+\int_{T_{s} / 2}^{\infty}\left(\left\{h^{0}, h^{1}\right\}\left(x^{\mathrm{s}}, t / \varepsilon\right)-\left\{h^{0}, h^{1}\right\}\left(\gamma_{p}, t / \varepsilon\right)\right) \mathrm{d} t\right],
\end{array}
$$


where $x^{\mathrm{u}}, x^{\mathrm{s}}$ and $\gamma_{p}$ denote, respectively, $x^{\mathrm{u}}(t, s), x^{\mathrm{s}}(t, s)$ and $\gamma_{p}(t / \varepsilon)$. Adding and subtracting the Melnikov integral, we get:

$$
\begin{aligned}
h^{0}\left(x^{\mathrm{u}}\left(T_{s} / 2, s\right)\right. & -h^{0}\left(x^{\mathrm{s}}\left(T_{s} / 2, s\right)\right) \\
= & \mu \varepsilon^{p}\left[\int_{-\infty}^{-T_{s} / 2}\left(\left\{h^{0}, h^{1}\right\}\left(x^{\mathrm{u}}, t / \varepsilon\right)-\left\{h^{0}, h^{1}\right\}\left(\gamma_{p}, t / \varepsilon\right)-\left\{h^{0}, h^{1}\right\}\left(x^{0}, t / \varepsilon\right)\right) \mathrm{d} t\right. \\
& +\int_{-T_{s} / 2}^{T_{s} / 2}\left(\left\{h^{0}, h^{1}\right\}\left(x^{\mathrm{u}}, t / \varepsilon\right)-\left\{h^{0}, h^{1}\right\}\left(\gamma_{p}, t / \varepsilon\right)-\left\{h^{0}, h^{1}\right\}\left(x^{0}, t / \varepsilon\right)\right) \mathrm{d} t \\
& +\int_{T_{s} / 2}^{+\infty}\left(\left\{h^{0}, h^{1}\right\}\left(x^{\mathrm{s}}, t / \varepsilon\right)-\left\{h^{0}, h^{1}\right\}\left(\gamma_{p}, t / \varepsilon\right)-\left\{h^{0}, h^{1}\right\}\left(x^{0}, t / \varepsilon\right)\right) \mathrm{d} t \\
& \left.+\int_{-\infty}^{+\infty}\left\{h^{0}, h^{1}\right\}\left(x^{0}, t / \varepsilon\right) \mathrm{d} t\right],
\end{aligned}
$$

where $x^{0}$ denotes $x^{0}(t+s)$ inside the four integrals in this expression. The last one is the Melnikov function (1.4) and we have to bound the other three integrals. The first and the third ones are $\mathrm{O}\left(\mu \varepsilon^{p+1}\right)$ by $(2.10)$. For the second one we need the following lemma:

Lemma 5.1 For $s \in \mathbf{C},|\Im s| \leq a-\varepsilon$ and $T_{s}:=T-\Re s$,

$$
\int_{-T_{s} / 2}^{T_{s} / 2}\left|\left\{h^{0}, h^{1}\right\}\left(x^{\mathrm{u}}, t / \varepsilon\right)-\left\{h^{0}, h^{1}\right\}\left(\gamma_{p}, t / \varepsilon\right)-\left\{h^{0}, h^{1}\right\}\left(x^{0}, t / \varepsilon\right)\right| \mathrm{d} t \leq K \mu \varepsilon^{\gamma-\ell+r-1},
$$

where $x^{\mathrm{u}}, x^{0}$ and $\gamma_{p}$ denote, respectively, $x^{\mathrm{u}}(t, s), x^{0}(t+s)$ and $\gamma_{p}(t / \varepsilon)$.

Proof. Since $\left\{h^{0}, h^{1}\right\}(x, t / \varepsilon)=-f\left(x_{1}\right) \partial_{2} h^{1}(x, t / \varepsilon)-x_{2} \partial_{1} h^{1}(x, t / \varepsilon)$, we can write

$$
\begin{aligned}
\left\{h^{0}, h^{1}\right\} & \left(x^{\mathrm{u}}, t / \varepsilon\right)-\left\{h^{0}, h^{1}\right\}\left(x^{0}, t / \varepsilon\right) \\
= & -f\left(x_{1}^{\mathrm{u}}\right)\left(\partial_{2} h^{1}\left(x^{\mathrm{u}}, t / \varepsilon\right)-\partial_{2} h^{1}\left(x^{0}, t / \varepsilon\right)\right)-\left(f\left(x_{1}^{\mathrm{u}}\right)-f\left(x_{1}^{0}\right)\right) \partial_{2} h^{1}\left(x^{0}, t / \varepsilon\right) \\
& -x_{2}^{\mathrm{u}}\left(\partial_{1} h^{1}\left(x^{\mathrm{u}}, t / \varepsilon\right)-\partial_{1} h^{1}\left(x^{0}, t / \varepsilon\right)\right)-\left(x_{2}^{\mathrm{u}}-x_{2}^{0}\right) \partial_{1} h^{1}\left(x^{0}, t / \varepsilon\right) .
\end{aligned}
$$

With the help of bounds (4.13), (4.14) and Lemma 4.2, we now proceed to estimate this expression. Using also the bound $|\xi(t)|_{\tau} \leq K \mu \varepsilon^{\gamma}$, which comes from the estimate $\xi:=x^{\mathrm{u}}-x^{0}=\mathrm{O}\left(\mu \varepsilon^{\gamma}\right)$, we get

$$
\begin{aligned}
& \left|\left\{h^{0}, h^{1}\right\}\left(x^{\mathrm{u}}(t, s), t / \varepsilon\right)-\left\{h^{0}, h^{1}\right\}\left(x^{0}(t+s), t / \varepsilon\right)\right| \\
& \leq\left(\frac{K}{\tau^{r+1}}+\frac{\mu \varepsilon^{\gamma}}{\tau^{2}}\right) \frac{\mu \varepsilon^{\gamma}}{\tau^{\ell-2 r+1}}+\frac{\mu \varepsilon^{\gamma}}{\tau^{2}} \frac{K}{\tau^{\ell-r}}+\left(\frac{K}{\tau^{r}}+\mu \varepsilon^{\gamma}\right) \frac{\mu \varepsilon^{\gamma}}{\tau^{\ell-2 r+2}}+\mu \varepsilon^{\gamma} \frac{K}{\tau^{\ell-r+1}} \\
& \leq K \frac{\mu \varepsilon^{\gamma}}{\tau^{\ell-r+2}} .
\end{aligned}
$$

Applying (4.16) with $\beta=\ell-r+2$, it turns out that the integral of the expression above is $\mathrm{O}\left(\mu \varepsilon^{\gamma-\ell+r-1}\right)$. The integral involving $\gamma_{p}$ is $\mathrm{O}\left(\mu \varepsilon^{p+1}\right)$, as $\gamma_{p}=\mathrm{O}\left(\mu \varepsilon^{p+1}\right)$ (see 
the Normal Form Theorem), and $f(0)=0$, hence $\left\{h^{0}, h^{1}\right\}\left(\gamma_{p}, t / \varepsilon\right)=\mathrm{O}\left(\mu \varepsilon^{p+1}\right)$, and the same will happen to its integral over $\left[-T_{s} / 2, T_{s} / 2\right]$.

Collecting the bounds for the three integrals, we obtain

$$
h^{0}\left(x^{\mathrm{u}}\left(T_{s} / 2, s\right)\right)-h^{0}\left(x^{\mathrm{s}}\left(T_{s} / 2, s\right)\right)=\mu \varepsilon^{p} M(s, \varepsilon)+\mathrm{O}\left(\mu \varepsilon^{p} \mu \varepsilon^{\gamma-\ell+r-1}, \mu^{2} \varepsilon^{2 p+1}\right),
$$

and thus (2.21) follows from (5.1).

2. We can take advantage of estimate (2.21) on complex values of $s$ to simply estimate the Fourier coefficients $\mathcal{E}_{k}^{\mathrm{u}}$ of $\mathcal{E}^{\mathrm{u}}(s)=\sum_{k} \mathcal{E}_{k}^{\mathrm{u}} \mathrm{e}^{\mathrm{i} k s / \varepsilon}$, as is standard in Cauchy bounds. For $k \neq 0$, we shift along complex lines $\Im u= \pm r_{\varepsilon}$, with $r_{\varepsilon}=a-\varepsilon$ to obtain:

$$
\mathcal{E}_{k}^{\mathrm{u}}=\frac{\mathrm{e}^{-|k| r_{\varepsilon} / \varepsilon}}{2 \pi \varepsilon} \int_{0}^{2 \pi \varepsilon} \mathcal{E}^{\mathrm{u}}\left(\sigma \pm \mathrm{i} r_{\varepsilon}\right) \mathrm{e}^{\mathrm{i} k \sigma / \varepsilon} \mathrm{d} \sigma=\mu \varepsilon^{p} M_{k}(\varepsilon)+\mathrm{O}\left(\mu^{2} \varepsilon^{2 \gamma+r-1}, \mu \varepsilon^{p+1}\right) \mathrm{e}^{-|k| a / \varepsilon}
$$

where $M_{k}(\varepsilon)$ are the Fourier coefficients of the Melnikov function. Now, estimate (2.22) follows readily.

3. We recall that $\mathcal{S}^{\mathrm{u}}(s)-s$ is a $2 \pi \varepsilon$-periodic, analytic function, defined on $|\Im s| \leq a-\varepsilon$ by $\mathcal{S}^{\mathrm{u}}(s)-s=\mathcal{S}\left(x^{\mathrm{u}}(t, s), t / \varepsilon\right)-t-s$, for any $t \in \mathbf{R}$ such that $T / 2 \leq t+\Re s \leq T$. By $(2.13), \mathcal{S}$ is $\mathrm{O}\left(\mu \varepsilon^{p+1}\right)$-close to $\mathcal{S}^{0}$, which in its turn satisfies $\mathcal{S}^{0}\left(x^{0}(t+s)\right)=t+s$ as an special case of (2.15). Using also the estimate (2.18) provided by the Extension Theorem, we arrive at

$$
\begin{aligned}
\mathcal{S}^{\mathrm{u}}(s)-s & =\mathcal{S}\left(x^{\mathrm{u}}(t, s), t / \varepsilon\right)-t-s=\mathcal{S}^{0}\left(x^{\mathrm{u}}(t, s)\right)-t-s+\mathrm{O}\left(\mu \varepsilon^{p+1}\right) \\
& =\mathcal{S}^{0}\left(x^{0}(t+s)\right)-t-s+\mathrm{O}\left(\mu \varepsilon^{\gamma}, \mu \varepsilon^{p+1}\right)=\mathrm{O}\left(\mu \varepsilon^{\gamma}\right)
\end{aligned}
$$

for $|\Im s| \leq a-\varepsilon$. Estimating now the Fourier coefficients of $\mathcal{S}^{\mathrm{u}}(s)-s$ and also those of its derivative (which has zero mean), we get, for real values of $s$,

$$
\begin{aligned}
\mathcal{S}^{\mathrm{u}}(s)-s & =\mathcal{S}_{0}^{\mathrm{u}}+\mathrm{O}\left(\mu \varepsilon^{\gamma}\right) \mathrm{e}^{-a / \varepsilon} \\
\frac{\mathrm{d} \mathcal{S}^{\mathrm{u}}}{\mathrm{d} s}(s)-1 & =\mathrm{O}\left(\mu \varepsilon^{\gamma-1}\right) \mathrm{e}^{-a / \varepsilon}
\end{aligned}
$$

and therefore $\mathcal{S}^{\mathrm{u}}(s)$ can be inverted for real $s$. (In fact, it can also be inverted for $|\Im s| \leq a-\varepsilon|\ln \varepsilon|$.

For real $s$, we can bound $\mathcal{S}^{\mathrm{u}}(s)-s$ repeating the computations above, but using estimate (2.19) provided by the real version of the Extension Theorem, instead of (2.18). This gives

$$
\mathcal{S}^{\mathrm{u}}(s)=s+\mathrm{O}\left(\mu \varepsilon^{p+1}\right)
$$

and in particular $\mathcal{S}_{0}^{\mathrm{u}}=\mathrm{O}\left(\mu \varepsilon^{p+1}\right)$ for the zero order Fourier coefficient $\mathcal{S}_{0}^{\mathrm{u}}$ of $\mathcal{S}^{\mathrm{u}}(s)-s$ that appears in formula (5.3).

Using formulae (5.2) and (5.3), we obtain for the inverse function $s=s^{\mathrm{u}}(S)$ of $S=\mathcal{S}^{\mathrm{u}}(s)$ that $s^{\mathrm{u}}(S)-S$ is $\mathrm{O}\left(\mu \varepsilon^{p+1}\right)$ and a $2 \pi \varepsilon$-periodic, analytic function. 


\section{Proof of Proposition 2.7}

It is clear that $\psi$ is an analytic and periodic function because so are $\mathcal{E}^{\mathrm{u}}$ and $\mathcal{S}^{\mathrm{u}}-s$ given in $(2.20)$.

1. For real $s$, according to property (2) of Corollary 2.2 , the invariant stable and unstable curves $C^{*}, *=\mathrm{s}$, u for the Poincaré map $P$ defined in (1.3) are given by $C^{*}=\left\{x^{*}(0, s)\right\}$, and the dynamics on them is simply given by a shift in the variable $s$ : $P\left(x^{*}(0, s)\right)=x^{*}(2 \pi \varepsilon, s)=x^{*}(0, s+2 \pi \varepsilon)$. Since the Poincaré map $P$ is area preserving, $C^{\mathrm{s}}$ and $C^{\mathrm{u}}$ must intersect at least in a homoclinic point $x^{h}=x^{\mathrm{u}}\left(0, h^{\mathrm{u}}\right)=x^{\mathrm{s}}\left(0, h^{\mathrm{s}}\right)$, giving rise to a homoclinic orbit: $x^{\mathrm{u}}\left(t, h^{\mathrm{u}}\right)=x^{\mathrm{s}}\left(t, h^{\mathrm{s}}\right), \forall t \in \mathbf{R}$. To see that $h^{\mathrm{s}}=\mathcal{S}^{\mathrm{u}}\left(h^{\mathrm{u}}\right)$, we take $t$ big enough $(t+s \geq T / 2)$ in such a way that $x^{\mathrm{s}}\left(t, h^{\mathrm{s}}\right) \in D^{\mathrm{s}}$, and then equations (2.15) for $s=h^{\mathrm{s}}$ read as

$$
\mathcal{S}\left(x^{\mathrm{s}}\left(t, h^{\mathrm{s}}\right), t / \varepsilon\right)-t=h^{\mathrm{s}}, \quad \mathcal{E}\left(x^{\mathrm{s}}\left(t, h^{\mathrm{s}}\right), t / \varepsilon\right)=0,
$$

and from the first equality above and definition $(2.20)$ of $\mathcal{S}^{\mathrm{u}}$, we get

$$
h^{\mathrm{s}}=\mathcal{S}\left(x^{\mathrm{s}}\left(t, h^{\mathrm{s}}\right), t / \varepsilon\right)-t=\mathcal{S}\left(x^{\mathrm{u}}\left(t, h^{\mathrm{u}}\right), t / \varepsilon\right)-t=\mathcal{S}^{\mathrm{u}}\left(h^{\mathrm{u}}\right),
$$

as wanted. Now, from the definition (2.23) of $\psi$, as well as (2.20) and (5.4),

$$
\psi\left(h^{\mathrm{s}}\right)=\psi\left(\mathcal{S}^{\mathrm{u}}\left(h^{\mathrm{u}}\right)\right)=\mathcal{E}^{\mathrm{u}}\left(h^{\mathrm{u}}\right)=\mathcal{E}\left(x^{\mathrm{u}}\left(t, h^{\mathrm{u}}\right), t / \varepsilon\right)=\mathcal{E}\left(x^{\mathrm{s}}\left(t, h^{\mathrm{s}}\right), t / \varepsilon\right)=0,
$$

and by the $2 \pi \varepsilon$-periodicity of $\psi, \psi\left(h_{n}\right)=0$ also holds.

To compute $\psi^{\prime}\left(h_{n}\right)=\psi^{\prime}\left(h^{\mathrm{s}}\right)$ we differentiate $(2.24)$ at the point $h^{\mathrm{s}}$, obtaining

$$
\psi^{\prime}\left(h^{\mathrm{s}}\right)=\partial_{1} \mathcal{E}\left(\tilde{x}^{\mathrm{u}}\left(t, h^{\mathrm{s}}\right), t / \varepsilon\right) \cdot \frac{\partial \tilde{x}_{1}^{\mathrm{u}}}{\partial S}\left(t, h^{\mathrm{s}}\right)+\partial_{2} \mathcal{E}\left(\tilde{x}^{\mathrm{u}}\left(t, h^{\mathrm{s}}\right), t / \varepsilon\right) \cdot \frac{\partial \tilde{x}_{2}^{\mathrm{u}}}{\partial S}\left(t, h^{\mathrm{s}}\right) .
$$

Finally, differentiating both equations (2.15) with respect to $s=S$, and taking into account that change (2.11) is canonical we get

$$
\begin{aligned}
& \frac{\partial x_{1}^{\mathrm{s}}}{\partial S}\left(t, h^{\mathrm{s}}\right)=\partial_{2} \mathcal{E}\left(x^{\mathrm{s}}\left(t, h^{\mathrm{s}}\right), t / \varepsilon\right)=\partial_{2} \mathcal{E}\left(\tilde{x}^{\mathrm{u}}\left(t, h^{\mathrm{s}}\right), t / \varepsilon\right), \\
& \frac{\partial x_{2}^{\mathrm{s}}}{\partial S}\left(t, h^{\mathrm{s}}\right)=-\partial_{1} \mathcal{E}\left(x^{\mathrm{s}}\left(t, h^{\mathrm{s}}\right), t / \varepsilon\right)=-\partial_{1} \mathcal{E}\left(\tilde{x}^{\mathrm{u}}\left(t, h^{\mathrm{s}}\right), t / \varepsilon\right),
\end{aligned}
$$

and inserting these equalities in the formula of $\psi^{\prime}\left(h_{n}\right)$, we get the required expression:

$$
\psi^{\prime}\left(h_{n}\right)=-\frac{\partial x_{2}^{\mathrm{s}}}{\partial S}\left(t, h^{\mathrm{s}}\right) \cdot \frac{\partial \tilde{x}_{1}^{\mathrm{u}}}{\partial S}\left(t, h^{\mathrm{s}}\right)+\frac{\partial x_{1}^{\mathrm{s}}}{\partial S}\left(t, h^{\mathrm{s}}\right) \cdot \frac{\partial \tilde{x}_{2}^{\mathrm{u}}}{\partial S}\left(t, h^{\mathrm{s}}\right) .
$$

Since the formula above for $\psi^{\prime}\left(h_{n}\right)$ is independent of $n$, it can be used to compute the angle at any homoclinic point $x^{\mathrm{s}}\left(0, h_{n}\right)=x^{\mathrm{s}}\left(2 \pi \varepsilon n, h^{\mathrm{s}}\right)$.

2. The points $x^{h}=x^{\mathrm{s}}\left(0, h^{\mathrm{s}}\right)$ and $P\left(x^{h}\right)=x^{\mathrm{s}}\left(0, h^{\mathrm{s}}+2 \pi \varepsilon\right)$ are homoclinic points of $P$, but since $P$ is orientation preserving there is another first homoclinic point between them: $x^{\mathrm{s}}\left(0, \bar{h}^{\mathrm{s}}\right)=x^{\mathrm{u}}\left(0, \bar{h}^{\mathrm{u}}\right)=\tilde{x}^{\mathrm{u}}\left(0, \bar{h}^{\mathrm{s}}\right)$, where $h^{\mathrm{s}}$ and $\bar{h}^{\mathrm{s}}$ are two consecutive zeros of $\psi(S)$. 
Since $P$ is area preserving, the area $A$ of the lobe $D$ in coordinates $x$ is the same as the area of the lobe $D^{\prime}$ in coordinates $(S, E)$, and can be computed at any image of the homoclinic points, giving rise to the desired formula

$$
A=\iint_{D^{\prime}} \mathrm{d} S \mathrm{~d} E=\left|\int_{h^{\mathrm{s}}}^{\bar{h}^{\mathrm{s}}} \psi(S) \mathrm{d} S\right| .
$$

3. In order to see that $\psi_{0}=0$, we only need to check that the area of the inner lobes and the outer lobes is the same. This can be easily seen making one iterate of the map $P$ and using the fact that the total area between the global invariant manifolds is invariant.

4. By the definition of $\psi$, and applying (2.22), we have for real $S$

$$
\psi(S)=\mathcal{E}_{0}^{\mathrm{u}}+\mu \varepsilon^{p} M\left(s^{\mathrm{u}}(S), \varepsilon\right)+\mathrm{O}\left(\mu^{2} \varepsilon^{2 \gamma+r-1}, \mu \varepsilon^{p+1}\right) \mathrm{e}^{-a / \varepsilon} .
$$

An straightforward computation of the Melnikov function shows that

$$
\mu \varepsilon^{p} M^{\prime}(S, \varepsilon)=\mathrm{O}\left(\mu \varepsilon^{\gamma-1} \mathrm{e}^{-a / \varepsilon}\right),
$$

for real $S$. Applying Taylor's Theorem to $\mu \varepsilon^{p} M\left(s^{\mathrm{u}}(S), \varepsilon\right)=\mu \varepsilon^{p} M\left(S+\mathrm{O}\left(\mu \varepsilon^{p+1}\right), \varepsilon\right)$, we get

$$
\begin{aligned}
\mu \varepsilon^{p} M\left(s^{\mathrm{u}}(S), \varepsilon\right) & =\mu \varepsilon^{p} M(S, \varepsilon)+\mathrm{O}\left(\mu \varepsilon^{\gamma-1} \mathrm{e}^{-a / \varepsilon} \mu \varepsilon^{p+1}\right) \\
& =\mu \varepsilon^{p} M(S, \varepsilon)+\mathrm{O}\left(\mu^{2} \varepsilon^{\gamma+p}\right) \mathrm{e}^{-a / \varepsilon}
\end{aligned}
$$

and then, since $\ell \geq r-1$, we have that $\gamma+p \geq 2 \gamma+r-1$, and

$$
\psi(S)=\mathcal{E}_{0}^{\mathrm{u}}+\mu \varepsilon^{p} M(S, \varepsilon)+\mathrm{O}\left(\mu^{2} \varepsilon^{2 \gamma+r-1}, \mu \varepsilon^{p+1}\right) \mathrm{e}^{-a / \varepsilon} .
$$

Now, since $\psi_{0}=0$, we obtain that $\mathcal{E}_{0}^{\mathrm{u}}=\mathrm{O}\left(\mu^{2} \varepsilon^{2 \gamma+r-1}, \mu \varepsilon^{p+1}\right) \mathrm{e}^{-a / \varepsilon}$, which gives equation (2.25).

To check estimate (5.6), we simply apply residues theory to compute the Fourier coefficients $M_{n}(\varepsilon)$ of the Melnikov function $M(s, \varepsilon)=\sum_{n \neq 0} M_{n}(\varepsilon) \mathrm{e}^{\mathrm{i} n t / \varepsilon}$ defined in (1.4) in terms of the Poisson bracket $J(x, t / \varepsilon)=\left\{h^{0}, h^{1}\right\}(x, t / \varepsilon)$, which is a $2 \pi \varepsilon$-periodic in $t$ function with zero mean. We now relate its Fourier series in the $\theta$ variable $J(x, \theta)=$ $\sum_{n \neq 0} J_{n}(x) \mathrm{e}^{\mathrm{i} n \theta}$, with the Fourier series of the Melnikov function

$$
\begin{aligned}
\mu \varepsilon^{p} M(s, \varepsilon) & =\mu \varepsilon^{p} \int_{-\infty}^{\infty} J\left(x^{0}(t+s), t / \varepsilon\right) \mathrm{d} t=\mu \varepsilon^{p} \int_{-\infty}^{\infty} J\left(x^{0}(u), \frac{u-s}{\varepsilon}\right) \mathrm{d} u \\
& =\mu \varepsilon^{p} \sum_{n \neq 0} \mathrm{e}^{-\mathrm{i} n s / \varepsilon} \int_{-\infty}^{\infty} J_{n}\left(x^{0}(u)\right) \mathrm{e}^{\mathrm{i} n u / \varepsilon} \mathrm{d} u=\mu \varepsilon^{p} \sum_{n \neq 0} \mathrm{e}^{-\mathrm{i} n s / \varepsilon} M_{-n}(\varepsilon),
\end{aligned}
$$

obtaining thus $M_{n}(\varepsilon)=\int_{-\infty}^{\infty} J_{-n}\left(x^{0}(u)\right) \mathrm{e}^{-\mathrm{i} n u / \varepsilon} \mathrm{d} u$.

Let us recall here that by hypothesis $H 2, x^{0}(u)$ is analytic on the strip $|\Im u|<a$, with only one isolated singularity on each line $\Im u= \pm a$. To compute $M_{n}$ we change the integration path down to $\Im u=-b$, if $n>0$, and up to $\Im u=b$, if $n<0$; with 
$b>a$ such that $x^{0}(u)$ has no more singularities on $|\Im u| \leq b$. Then, taking into account that $u \mapsto J\left(x^{0}(u), \theta\right)$ has a pole of order $\leq \ell+1$ at $u= \pm a$ i it follows that $J_{n}\left(x^{0}(u)\right)$ has at most a pole of this order at $u= \pm a$ i, i.e., its Laurent series has the form $J_{n}\left(x^{0}(u)\right)=\sum_{k \leq \ell+1} J_{n, k}(u-a \mathrm{i})^{-k}$. Applying residues theory, we obtain

$$
\mu \varepsilon^{p} M_{n}+\mathrm{O}\left(\mathrm{e}^{-n b / \varepsilon}\right)=\mu \varepsilon^{p-\ell} \mathrm{e}^{-n a / \varepsilon}\left[(-\mathrm{i} n)^{\ell} J_{-n, \ell+1}+\mathrm{O}(\varepsilon)\right],
$$

for instance for $n>0$. The $\mathrm{O}\left(\mathrm{e}^{-n b / \varepsilon}\right)$ term comes from the integral through the path parallel to the real axis, with $\Im u=-b<-a$. We can conclude that, for $n>0$,

$$
\begin{aligned}
\mu \varepsilon^{p} M_{n} & =\mu \varepsilon^{\gamma} \mathrm{e}^{-n a / \varepsilon}\left[(-\mathrm{i} n)^{\ell} J_{-n, \ell+1}+\mathrm{O}(\varepsilon)\right] \\
& =\mu \varepsilon^{\gamma} \mathrm{e}^{-n a / \varepsilon} \tilde{M}_{n},
\end{aligned}
$$

and an analogous formula for $n<0$

$$
\begin{aligned}
\mu \varepsilon^{p} M_{n} & =\mu \varepsilon^{\gamma} \mathrm{e}^{-n a / \varepsilon}\left[(-\mathrm{i} n)^{\ell} J_{-n, \ell+1}+\mathrm{O}(\varepsilon)\right] \\
& =\mu \varepsilon^{\gamma} \mathrm{e}^{-n a / \varepsilon} \tilde{M}_{n},
\end{aligned}
$$

with $\tilde{M}_{n}=\mathrm{O}(1)$. In conclusion, from formulae (5.7), (5.8), we obtain $\mu \varepsilon^{p} M_{k}(\varepsilon)=$ $\mathrm{O}\left(\mu \varepsilon^{\gamma} \mathrm{e}^{-|k| a / \varepsilon}\right)$, and thus estimate (5.6) follows.

\section{Proof of Corollary 1.2}

First of all, formulae (5.8), and (5.7), give us

$$
\begin{aligned}
\mu \varepsilon^{p} M(s, \varepsilon) & =\mu \varepsilon^{\gamma} \sum_{n>0} \mathrm{e}^{-n a / \varepsilon}\left(\tilde{M}_{-n} \mathrm{e}^{-\mathrm{i} n s / \varepsilon}+\tilde{M}_{n} \mathrm{e}^{\mathrm{i} n s / \varepsilon}\right) \\
& =\mu \varepsilon^{p} \mathrm{e}^{-a / \varepsilon}\left[\tilde{M}_{-1}(\varepsilon) \mathrm{e}^{-\mathrm{i} s / \varepsilon}+\tilde{M}_{1}(\varepsilon) \mathrm{e}^{\mathrm{i} s / \varepsilon}\right]+\mathrm{O}\left(\mathrm{e}^{-2 a / \varepsilon}\right) \\
& =\mu \varepsilon^{p}\left[M_{-1}(\varepsilon) \mathrm{e}^{-\mathrm{i} s / \varepsilon}+M_{1}(\varepsilon) \mathrm{e}^{\mathrm{i} s / \varepsilon}\right]+\mathrm{O}\left(\mathrm{e}^{-2 a / \varepsilon}\right),
\end{aligned}
$$

and it only remains to check $\mu \varepsilon^{p} M_{ \pm 1} \sim \mu \varepsilon^{\gamma} \mathrm{e}^{-a / \varepsilon}$. Again from formulae (5.7) and (5.8) for $n= \pm 1$, we get

$$
\left.\mu \varepsilon^{p}\left[M_{-1} \mathrm{e}^{-i s / \varepsilon}+M_{1} \mathrm{e}^{\mathrm{i} s / \varepsilon}\right]=\mu \varepsilon^{\gamma} \mathrm{e}^{-a / \varepsilon}\left(\mathrm{i}^{\ell} J_{1, \ell+1} \mathrm{e}^{-\mathrm{i} s / \varepsilon}+(-\mathrm{i})^{\ell} J_{-1, \ell+1} \mathrm{e}^{+\mathrm{i} s / \varepsilon}+\mathrm{O}(\varepsilon)\right)\right) .
$$

From hypothesis $H 4$, we know that $J_{1, \ell+1}=\overline{J_{-1, \ell+1}}$ are not zero. Writing $J_{1, \ell+1}=$ $\left|J_{1, \ell+1}\right| \mathrm{e}^{\mathrm{i} \varphi}, J_{-1, \ell+1}=\left|J_{1, \ell+1}\right| \mathrm{e}^{-\mathrm{i} \varphi}$, with $\left|J_{1, \ell+1}\right|>0$, we obtain that

$$
\mu \varepsilon^{p} M(s, \varepsilon)=2\left|J_{1, \ell+1}\right|(-1)^{\ell} \mu \varepsilon^{\gamma} \mathrm{e}^{-a / \varepsilon} \cos (s / \varepsilon-\varphi)+\mathrm{O}\left(\mathrm{e}^{-2 a / \varepsilon}\right),
$$

and putting this expression into the formulae of the area and the angle given by Theorem 1.1 we obtain the desired result. 


\section{Acknowledgments}

This work has been partially supported by the Spanish grant DGICYT PB94-0215, the EC grant ERBCHRXCT940460, and the Catalan grant CIRIT GRQ93-1135. Both authors are indebted to C. Simó, and V. Gelfreich for useful comments, and specially to $R$. de la Llave for fruitful discussions and remarks about previous versions of this paper. This work was finished while the first author was a visitor at the Institute for Mathematics and Its Applications in Minneapolis, for whose hospitality he is very grateful.

\section{References}

[Ang93] S. Angenent. A variational interpretation of Melnikov's function and exponentially small separatrix splitting. In D. Salamon, editor, Symplectic geometry, volume 192 of London Math. Soc. Lecture Note Ser., pages 5-35. Cambridge Univ. Press, 1993.

[CG94] L. Chierchia and G. Gallavotti. Drift and diffusion in phase space. Ann. Inst. H. Poincaré Phys. Théor., 60(1):1-144, 1994.

[DGJS96] A. Delshams, V.G. Gelfreich, A. Jorba, and T.M. Seara. Exponentially small splitting of separatrices under fast quasiperiodic forcing. Math. Preprints Series 199, Univ. Barcelona, February 1996. To appear in Comm. Math. Phys.

[DGJS97] A. Delshams, V.G. Gelfreich, A. Jorba, and T.M. Seara. Lower and upper bounds for the splitting of separatrices of the pendulum under a fast quasiperiodic forcing. ERA Amer. Math. Soc., 3:1-10, 1997.

[DR96] A. Delshams and R. Ramírez-Ros. Poincaré-Melnikov-Arnold method for analytic planar maps. Nonlinearity, 9(1):1-26, 1996.

[DS92] A. Delshams and T.M. Seara. An asymptotic expression for the splitting of separatrices of the rapidly forced pendulum. Comm. Math. Phys., 150:433-463, 1992.

[DS93] A. Delshams and T.M. Seara. Exponentially small expressions for separatrix splittings. In Kuksin et al. [KLP93], pages 68-80.

[DS94] A. Delshams and T.M. Seara. Exponentially small splitting in Hamiltonian systems. In Seimenis [Sei94], pages 181-187.

[EKS93] J.A. Ellison, M. Kummer, and A.W. Sáenz. Transcendentally small transversality in the rapidly forced pendulum. J. Dynamics Differential Equations, 5(2):241-277, 1993.

[Fon93] E. Fontich. Exponentially small upper bounds for the splitting of separatrices for high frequency periodic perturbations. Nonlinear Anal., 20(6):733-744, 1993.

[Fon95] E. Fontich. Rapidly forced planar vector fields and splitting of separatrices. $J$. Differential Equations, 119(2):310-335, 1995. 
[FS96] B. Fiedler and J. Scheurle. Discretization of homoclinic orbits, rapid forcing and "invisible" chaos. Mem. Amer. Math. Soc., 119(570), 1996. viii + 79 pp.

[Gal94] G. Gallavotti. Twistless KAM tori, quasi flat homoclinic intersections, and other cancellations in the perturbation series of certain completely integrable Hamiltonian systems. A review. Rev. Math. Phys., 6(3):343-411, 1994.

[Gel93] V.G. Gelfreich. Separatrices splitting for the rapidly forced pendulum. In Kuksin et al. [KLP93], pages 47-67.

[Gel95] V.G. Gelfreich. Melnikov method and exponentially small splitting of separatrices. Math. Preprints Series 190, Univ. Barcelona, December 1995.

[Gel97] V.G. Gelfreich. Reference systems for splitting of separatrices. Nonlinearity, 10(1):175-193, 1997.

[GLT91] V.G. Gelfreich, V.F. Lazutkin, and M.B. Tabanov. Exponentially small splitting in Hamiltonian systems. Chaos, 1(2):137-142, 1991.

[HMS88] P. Holmes, J. Marsden, and J. Scheurle. Exponentially small splittings of separatrices with applications to KAM theory and degenerate bifurcations. Contemp. Math., 81:213-244, 1988.

[KLP93] S. Kuksin, V.F. Lazutkin, and J. Pöschel, editors. Proceedings of the Dynamical Systems Semester. Held in St. Petersburg, Russia, 17-30 November, 1991. Birkhäuser, Basel-Boston-Stuttgart, 1993.

[Mos56] J. Moser. The analytic invariants of an area-preserving mapping near a hyperbolic fixed point. Comm. Pure Appl. Math., 9:673-692, 1956.

[Nei84] A.I. Neishtadt. The separation of motions in systems with rapidly rotating phase. J. Appl. Math. Mech., 48(2):133-139, 1984.

[Poi90] H. Poincaré. Sur le problème des trois corps et les équations de la dynamique. Acta Math., 13:1-271, 1890.

[RW97a] M. Rudnev and S. Wiggins. KAM theory near multiplicity one resonant surfaces in perturbations of a-priori stable Hamiltonian systems. J. Nonlinear Sci., 7:177-209, 1997.

[RW97b] M. Rudnev and S. Wiggins. Existence of exponentially small separatrix splittings and homoclinic connections between whiskered tori in weakly hyperbolic nearintegrable Hamiltonian systems. Preprint 97-4, mp_arc@math .utexas . edu, January 1997. To appear in Phys. D.

[Sau95] D. Sauzin. Résurgence paramétrique et exponentielle petitese de l'ecart des séparatrices du pendule rapidament forcé. Ann. Inst. Fourier (Grenoble), 45(2):453$511,1995$.

[Sei94] J. Seimenis, editor. Hamiltonian Mechanics: Integrability and Chaotic Behaviour, volume 331 of NATO Adv. Sci. Inst. Ser. B Phys. Held in Torun, Polland, 28 June2 July 1993. Plenum, New York, 1994. 
[Sim94] C. Simó. Averaging under fast quasiperiodic forcing. In Seimenis [Sei94], pages $13-34$.

[SMH91] J. Scheurle, J. Marsden, and P.J. Holmes. Exponentially small estimate for separatrix splittings. In H. Segur, S. Tanveer, and H. Levine, editors, Asymptotics beyond All Orders, pages 187-195. NATO Adv. Sci. Inst. Ser. B Phys., vol. 284, Plenum, New York, 1991.

[Tre97] D.V. Treschev. Separatrix splitting for a pendulum with rapidly oscillating suspension point. Russ. J. Math. Phys., 5(2):63-98, 1997.

\section{Internet access:}

All the authors' quoted preprints are available at http://www-ma1.upc.es in the Preprints pages, or at ftp://ftp-ma1.upc.es, in the pub/preprints directory.

E-mail addresses of the authors: amadeu@ma1.upc.es, tere@ma1.upc.es. 